\title{
ERICKSON'S CONJECTURE ON THE RATE OF ESCAPE OF $d$-DIMENSIONAL RANDOM WALK
}

BY

\section{HARRY KESTEN}

\begin{abstract}
We prove a strengthened form of a conjecture of Erickson to the effect that any genuinely $d$-dimensional random walk $S_{n}, d>3$, goes to infinity at least as fast as a simple random walk or Brownian motion in dimension $d$. More precisely, if $S_{n}^{*}$ is a simple random walk and $B_{t}$ a Brownian motion in dimension $d$, and $\psi:[1, \infty) \rightarrow(0, \infty)$ a function for which $t^{-1 / 2} \psi(t) \downarrow 0$, then $\psi(n)^{-1}\left|S_{n}^{*}\right| \rightarrow \infty$ w.p.1, or equivalently, $\psi(t)^{-1}\left|B_{t}\right|$ $\rightarrow \infty$ w.p.1, iff $\int_{1}^{\infty} \psi(t)^{d-2} t-d / 2<\infty$; if this is the case, then also $\psi(n)^{-1}\left|S_{n}\right| \rightarrow \infty$ w.p.1 for any random walk $S_{n}$ of dimension $d$.
\end{abstract}

1. Introduction. Let $X_{1}, X_{2}, \ldots$ be independent identically distributed random $d$-dimensional vectors and $S_{n}=\sum_{i=1}^{n} X_{i}$. Assume throughout that $d>3$ and that the distribution function $F$ of $X_{1}$ satisfies

$$
\operatorname{supp}(F) \text { is not contained in any hyperplane. }
$$

The celebrated Chung-Fuchs recurrence criterion [1, Theorem 6] implies that ${ }^{1}\left|S_{n}\right| \rightarrow \infty$ w.p.1 irrespective of $F$. In [4] Erickson made the much stronger conjecture that there should even exist a uniform escape rate for $S_{n}$, viz. that $n^{-\alpha}\left|S_{n}\right| \rightarrow \infty$ w.p.1 for all $\alpha<\frac{1}{2}$ and all $F$ satisfying (1.1). Erickson proved his conjecture in special cases and proved in all cases that $n^{-\alpha}\left|S_{n}\right| \rightarrow$ $\infty$ w.p.l for $\alpha<1 / 2-1 / d$. Our principal result is the following theorem which contains Erickson's conjecture and which makes precise the intuitive idea that a simple random walk $S_{n}^{*}$ (which corresponds to the distribution $F^{*}$ which puts mass $(2 d)^{-1}$ at each of the points $\left.(0,0, \ldots, \pm 1,0, \ldots, 0)\right)$ goes to infinity slower than any other random walk.

THEOREM. Let $d>3, S_{n}$ and $S_{n}^{*}$ as above, and let $\left\{B_{t}\right\}_{t>0}$ be a d-dimensional Brownian motion $\left(E B_{t} \equiv 0, E B_{t}(i) B_{t}(j)=t \delta_{i, j}\right)$. Assume that the function $\psi:[1, \infty) \rightarrow(0, \infty)$ satisfies

$$
t^{-1 / 2} \psi(t) \downarrow 0 \text {. }
$$

Then $\psi(n)^{-1}\left|S_{n}^{*}\right| \rightarrow \infty$ w.p.l and $\psi(t)^{-1}\left|B_{t}\right| \rightarrow \infty$ w.p.l are both equivalent to

Received by the editors June 25, 1976.

AMS (MOS) subject classifications (1970). Primary 60J15, 60F20.

Key words and phrases. Random walk, escape rate, concentration functions.

${ }^{1}$ For any vector $v \in \mathbf{R}^{d}$ we denote its components by $v(1), \ldots, v(d)$, and $|v|=$ $\left\{\sum_{i=1}^{d} v^{2}(i)\right\}^{1 / 2}$. "w.p.l" stands for "with probability one".

- American Mathematical Society 1978 


$$
\int_{1}^{\infty} \psi(t)^{d-2} t^{-d / 2}<\infty
$$

If (1.2) and (1.3) hold, then also $\psi(n)^{-1}\left|S_{n}\right| \rightarrow \infty$ w.p.1 whenever $F$ satisfies (1.1).

As we shall see this theorem is almost immediate from

Proposition 1. If $d \geqslant 3$ and $F$ satisfies (1.1) then there exist constants $0<\Gamma_{1}(F), \Gamma_{2}(F)<\infty$ such that for all $k \geqslant 1$ and all $A>0$,

$$
\begin{aligned}
P\left\{\left|S_{n}\right| \leqslant A\right. & \text { for some } \left.2^{k} \leqslant n<2^{k+1}\right\} \\
& <\Gamma_{1}(F)\left\{\left((A+1) 2^{-k / 2}\right)^{d-2}+\exp -\Gamma_{2}(F) k\right\} .
\end{aligned}
$$

It is worthwhile contrasting the theorem with the observation (see [4, end of $\left.\S 5]^{2}\right)$ that even if (1.1)-(1.3) hold, there may exist a deterministic sequence of vectors $a_{n}$ such that

$$
\liminf _{n \rightarrow \infty} \psi(n)^{-1}\left|S_{n}-a_{n}\right|=0 \quad \text { w.p. } 1 \text {. }
$$

Thus, the theorem is not merely a matter of estimating concentration functions for $S_{n}$. Nevertheless the result is intimately connected with concentration functions. Erickson's proof for $\psi(t)=t^{\alpha}, \alpha<1 / 2-1 / d$, uses a concentration function inequality of Esseen [6], and our proof makes heavy use of the following inequalities for concentration functions in dimension two. These estimates too are closely related to those of Esseen [5, Theorem 3], but are more generally applicable. We note that Corollary 1 shows that in the identically distributed case the concentration function decreases like $n^{-1}$.

Proposition 2. Let $Z_{1}, Z_{2}, \ldots, Z_{n}$ be independent random two-vectors with distribution functions $G_{1}, G_{2}, \ldots, G_{n}$. Let $\rho, \rho_{1}, \rho_{2}, \ldots, \rho_{n}$ be strictly positive numbers such that $\rho_{i}<\rho$, and let $A_{1}, A_{2}, \ldots, A_{n}$ be sets in $\mathbf{R}^{2} \times \mathbf{R}^{2}$ such that $A_{i} \subset\left\{u \in \mathbf{R}^{2}:|u|>\rho_{i}\right\}^{2}$. Define the symmetrization $G_{i}^{s}$ of $G_{i} b y^{3}$

and put $^{4}$

$$
G_{i}^{s}(B)=\int_{\mathbf{R}^{2}} G_{i}(B+u) d G_{i}(u)
$$

$$
\begin{gathered}
q_{i}=\int_{|u|>\rho_{i}} d G_{i}^{s}(u), \quad \sigma_{i}^{2}=\inf _{|\theta|=1} \int_{|u|<\rho_{i}}\langle\theta, u\rangle^{2} d G_{i}^{s}(u), \\
C=\sum_{i=1}^{n}\left\{\sigma_{i}^{2}+\frac{\rho_{i}^{2}}{q_{i}} \int_{u, v \in A_{i}} d G_{i}^{s}(u) d G_{i}^{s}(v)\right\} .
\end{gathered}
$$

\footnotetext{
${ }^{2}$ More details are given in the recent article by K. B. Erickson, Slowing down d-dimensional random walks, Ann. Probability 5 (1977), 645-651.

${ }^{3}$ For a distribution function $F$ and Borel set $A, F(A)$ denotes the mass assigned to $A$ by the Borel measure induced by $F$.

${ }^{4}\langle u, v\rangle$ denotes the usual inner product of two vectors of the same dimension.
} 
Finally, denote the angle between two vectors $u$ and $v$, determined in such a way that it lies in $[0, \pi]$, by $\varphi(u, v)$. Then there exists a universal constant $K_{1}<\infty$ such that

$$
\begin{aligned}
& \sup _{z \in \mathbf{R}^{2}} P\left\{\left|\sum_{1}^{n} Z_{i}+z\right|<\rho\right\} \\
& \leqslant \frac{K_{1} \rho^{2}}{C} \exp \left\{\sum_{i=1}^{n} \frac{\rho_{i}^{2}}{C q_{i}} \int_{u, v \in A_{i}} d G_{i}^{s}(u) d G_{i}^{s}(v)\right. \\
&\left.\qquad \log \left(1+\frac{\rho_{i}}{|v||\sin \varphi(u, v)|}\right)\right\} .
\end{aligned}
$$

COROllaRY 1. Let $Z_{1}, \ldots, Z_{n}$ be independent random two-vectors, all with the same distribution function $G$, and define $G^{s}$ as in (1.6). If $\rho>0$ and

$$
q=\int_{|u|>\rho} d G^{s}(u), \quad \sigma^{2}=\inf _{|\theta|=1} \int_{|u|<\rho}\langle\theta, u\rangle^{2} d G^{s}(u),
$$

and if there exist sets $B_{1}, B_{2} \subset\left\{z \in \mathbf{R}^{2}:|z|>\rho\right\}$ and a constant $c>0$ such that

$$
|v||\sin \varphi(u, v)| \geqslant c \quad \text { whenever } u \in B_{1}, v \in B_{2}
$$

then

$$
\begin{aligned}
& \sup _{z \in \mathbb{R}^{2}} P\left\{\left|\sum_{1}^{n} Z_{i}+z\right| \leqslant \rho\right\} \\
& \quad \leqslant \frac{K_{1}}{n} \rho^{2}\left(1+\rho c^{-1}\right)\left\{\sigma^{2}+\frac{\rho^{2}}{q} \int_{B_{1}} d G^{s}(u) \int_{B_{2}} d G^{s}(v)\right\}^{-1}
\end{aligned}
$$

Acknowledgement. We wish to thank Professor Erickson for making his results [4] available to us before publication and for some helpful conversations.

2. Reduction to a two dimensional problem. In this section we shall reduce the proof of Proposition 1 to a two dimensional problem. This problem will then be settled together with Proposition 2, by means of estimates on two dimensional concentration functions in $\$ 3$. Throughout $K_{1}, K_{2}, \ldots$ will be strictly positive constants which depend on the dimension $d$ only and whose numerical value is immaterial for our purposes. They will not necessarily have the same value at each appearance. $\Gamma_{1}, \Gamma_{2}, \ldots$ and $k_{1}, k_{2}, \ldots$ will be constants which depend on $F$ and $d$ or some other parameters; where necessary these parameters will be indicated explicitly, except that we shall not indicate dependence on $d$. For any random variable $Y$ we write $Y^{s}=Y-Y^{\prime}$ where 
$Y^{\prime}$ is independent of $Y$ and has the same distribution as $Y$. If $Y$ has distribution function $G$, then $Y^{s}$ has the distribution function $G^{s}$ given by

$$
G^{s}(B)=\int G(B+y) d G(y) .
$$

The first reduction shows that we may assume that $F$ has certain smoothness properties. It is of a purely technical nature and has little to do with the basic idea of the proof of Proposition 1. The reader should skip the proof of Lemma 1 at first reading.

Lemma 1. It suffices to prove Proposition 1 in the case where

$$
F(d x) \geqslant a d x \text { on } C_{0}
$$

for some $a>0$ and some closed cube $C_{0} \subset \mathbf{R}^{d}$ (which does not reduce to a point).

Proof. Let $F$ satisfy (1.1). We shall construct an $F_{1}$ which satisfies (2.1) (and a fortiori (1.1)) such that Proposition 1 holds for the original $S_{n}$ as soon as it holds for a random walk, $S_{n}\left(F_{1}\right)$ say, whose increments have distribution function $F_{1}$. For this purpose we first find a large cube

$$
C_{1}=\left\{z \in \mathbf{R}^{d}:-L \leqslant z(i) \leqslant L, 1<i<d\right\}
$$

such that $F\left(C_{1}\right)>0$ and such that the intersection of $\operatorname{supp}(F)$ with $C_{1}$ is not contained in any hyperplane. This is possible by (1.1). Define the distribution functions $G$ and $H$ by ${ }^{5}$

and

$$
p_{0}=\frac{1}{2} F\left(C_{1}\right), \quad G(S)=\left(2 p_{0}\right)^{-1} F\left(S \cap C_{1}\right)
$$

$$
H(S)=\left(1-p_{0}\right)^{-1}\left\{\frac{1}{2} F\left(S \cap C_{1}\right)+F\left(S \cap C_{1}^{c}\right)\right\} \text {. }
$$

Then we can write $F=p_{0} G+\left(1-p_{0}\right) H$, and if $I_{1}, I_{2}, \ldots, U_{1}, U_{2}, \ldots, V_{1}, V_{2}, \ldots$ are totally independent random variables with

$$
\begin{gathered}
P\left\{I_{j}=0\right\}=1-P\left\{I_{j}=1\right\}=p_{0}, \\
P\left\{U_{j} \in S\right\}=G(S), \quad P\left\{V_{j} \in S\right\}=H(S),
\end{gathered}
$$

then the joint distribution of $\left\{X_{n}\right\}_{n>1}$ is the same as of $\left\{U_{n}\left(1-I_{n}\right)+\right.$ $\left.V_{n} I_{n}\right\}_{n>1}$ (compare [9, p. 1184]). We shall therefore assume that $U, V$ and $I$ are defined on our original probability space and that $X_{n}=U_{n}\left(1-I_{n}\right)+$ $V_{n} I_{n}$. Let $\sigma_{1}<\sigma_{2}<\ldots$ be the successive (random) indices for which $I_{n}=1$. Then, with $\sigma_{0}=0$, the $\sigma_{i+1}-\sigma_{i}$ are independent and all with the distribution

$$
P\left\{\sigma_{i+1}-\sigma_{i}=r\right\}=p_{0}^{r-1}\left(1-p_{0}\right), \quad r \geqslant 1 .
$$

\footnotetext{
${ }^{5}$ For any set $S \subset \mathbf{R}^{d}, S^{c}$ denotes its complement, i.e., $\mathbf{R}^{d} \backslash S$.
} 
Even more, if $\mathscr{F}_{n}$ is the $\sigma$-field generated by $\left\{U_{j}, V_{j}, I_{j}: 1<j<n\right\}$, then for each $\left\{\mathscr{F}_{n}\right\}$ stopping time $T$ and

$$
\sigma^{*}(T)=\text { smallest } \sigma_{i} \text { which exceeds } T \text {, }
$$

one has

$$
P\left\{\sigma^{*}(T)=T+r \mid \mathscr{F}_{T}\right\}=p_{0}^{r-1}\left(1-p_{0}\right), \quad r>1 .
$$

It is also not hard to see that the $\left\{S_{\sigma_{i+1}}-S_{\sigma_{i}}\right\}_{i>0}$ are independent and all with the same distribution function ${ }^{6}$

$$
F_{2}=\sum_{r=1}^{\infty} P\left\{\sigma_{1}=r\right\} G^{*(r-1)} * H=\sum_{r=1}^{\infty} p_{0}^{r-1}\left(1-p_{0}\right) G^{*(r-1)} * H,
$$

and that also, conditional on $\mathscr{F}_{T}, S_{\sigma^{*}(T)}-S_{T}$ has the distribution $F_{2}$ on the set $\{T<\infty\}$. Now take

$$
T=\inf \left\{n \in\left[2^{k}, 2^{k+1}\right) \text { with }\left|S_{n}\right|<A\right\}
$$

(= $\infty$ if no such $T$ exists). Then, since $\left|S_{T}\right|<A$,

$$
\begin{aligned}
& P\left\{\left|S_{\sigma_{l}}\right| \leqslant 2 A \text { for some } l \in\left[\frac{1}{2}\left(1-p_{0}\right) 2^{k}, 2\left(1-p_{0}\right) 2^{k+2}\right)\right\} \\
&> P\left\{T<\infty,\left|S_{\sigma^{*}(T)}-S_{T}\right| \leqslant A\right\} \\
&-P\left\{T<\infty, \sigma^{*}(T)=\sigma_{l} \text { with } l \notin\left[\frac{1}{2}\left(1-p_{0}\right) 2^{k}, 2\left(1-p_{0}\right) 2^{k+2}\right)\right\} \\
&> P\{T<\infty\} F_{2}\left(\left\{z \in R^{d}:|z|<A\right\}\right) \\
&-P\left\{T<\infty, \sigma^{*}(T)-T>2^{k}\right\} \\
&-P\left\{\exists l \notin\left[\frac{1}{2}\left(1-p_{0}\right) 2^{k}, 2\left(1-p_{0}\right) 2^{k+2}\right) \text { with } \sigma_{l} \in\left[2^{k}, 2^{k+2}\right)\right\} .
\end{aligned}
$$

Now for some $A_{0}=A_{0}(F)<\infty$ and all $A>A_{0}$,

$$
F_{2}\left(\left\{z \in \mathbf{R}^{d}:|z|<A\right\}\right)>\frac{1}{2}, \quad P\left\{T<\infty, \sigma^{*}(T)-T>2^{k}\right\}<p_{0}^{2^{k}} .
$$

Also, with

$$
l_{1}=\left[\frac{1}{2}\left(1-p_{0}\right) 2^{k}\right], \quad l_{2}=\left[2\left(1-p_{0}\right) 2^{k+2}\right],
$$

the last term in (2.4) is at most

$$
P\left\{\sigma_{l_{1}} \geqslant 2^{k} \text { or } \sigma_{l_{2}}<2^{k+2}\right\} \leqslant \Gamma_{3} \exp -\Gamma_{4} 2^{k},
$$

for some $\Gamma_{3}, \Gamma_{4}<\infty$ depending on $p_{0}$ only (by (2.3) and standard exponential

\footnotetext{
${ }^{6} G * H$ denotes the convolution of $G$ and $H$, and $G^{*(s)}$ denotes the s-fold convolution of $G$ with itself.
} 
estimates; compare $[9$, formulae (5.40) $-(5.42)])$. Thus (2.4) yields for $A>$ $A_{0}$,

$$
\begin{aligned}
P\left\{\left|S_{n}\right|<A \text { for some } 2^{k}<n<2^{k+1}\right\}=P\{T<\infty\} \\
\quad<2 P\left\{\left|S_{\sigma_{l}}\right|<2 A \text { for some } l \in\left[\frac{1}{2}\left(1-p_{0}\right) 2^{k}, 2\left(1-p_{0}\right) 2^{k+2}\right)\right\} \\
\quad+2 p_{0}^{2^{k}}+2 \Gamma_{3} \exp -\Gamma_{4} 2^{k} .
\end{aligned}
$$

It is clear from (2.5) that if Proposition 1 holds for the random walk $S_{n}\left(F_{2}\right)=S_{\sigma_{n}}$, whose increments $S_{\sigma_{i+1}}-S_{\sigma_{i}}$ all have the distribution $F_{2}$, then Proposition 1 also holds for the original $S_{n}$. Actually (2.5) was derived only for $A \geqslant A_{0}$. However, for $A<A_{0}$ or even $A<2^{k / 8}$, (1.4) is immediate from Esseen's estimate [6, Corollary to Theorem 6.2]

$$
\begin{aligned}
P\left\{\left|S_{n}\right| \leqslant A\right\} & \leqslant K_{2}(A+1)^{d} \sup _{z} P\left\{\left|S_{n}+z\right| \leqslant 1\right\} \\
& \leqslant \Gamma_{5}(F)(A+1)^{d} n^{-d / 2}
\end{aligned}
$$

for some $\Gamma_{5}(F)<\infty$.

$F_{2}$ itself does not have to satisfy (2.1). However, set

$$
G_{2}=\sum_{r=1}^{\infty} p_{0}^{r-1}\left(1-p_{0}\right) G^{*(r-1)}
$$

so that $F_{2}=G_{2} * H$, and assume that we can find a distribution function $G_{1}$ with a continuous density such that

$$
\int_{\mathbf{R}^{d}} x^{\nu} d G_{1}(x)=\int_{\mathbf{R}^{d}} x^{\nu} d G_{2}(x) \text { for }\|\nu\|<16 .
$$

(Here we use the standard multi-index notation; $x^{p}=\prod_{i=1}^{d} x(i)^{\nu(i)}$, for positive integers $\nu(i)$ and $\|\nu\|=\sum_{i=1}^{d} \nu(i)$.) We claim that then $F_{1} \equiv G_{1} * H$ has the required properties. Before constructing $G_{1}$ we shall prove this claim. (2.1) is obvious for $F_{1}$; indeed $G_{1}$ has a continuous density and hence $F_{1}$ has a density which is lower semicontinuous. Thus we merely have to prove that the validity of Proposition 1 for $S_{n}\left(F_{1}\right)$ implies the validity of Proposition 1 for $S_{n}\left(F_{2}\right)$ (since we already showed that Proposition 1 then also holds for our original $S_{n}$ ). Now fix $k$ and $A \geqslant 2^{k / 8}$; we already saw above that (1.4) follows from (2.6) if $A<2^{k / 8}$ so that these are the only values of interest. Let $N$, $\chi_{1}, \chi_{2}, \ldots$ be independent random $d$-vectors, also independent of $\left\{S_{\sigma_{1}}\right\}_{1>0}$ and such that $N$ has a normal distribution with mean zero and covariance matrix $k^{-1} A^{2}$ times the identity matrix, and such that each $\chi_{i}$ has distribution $F_{1}$. Then, for any $n$, 


$$
\begin{aligned}
P\left\{\left|S_{\sigma_{n}}\right|\right. & <2 A\}<P\left\{\left|S_{o_{n}}(j)\right|<2 A, 1<j<d\right\} \\
& <P\left\{\left|S_{\sigma_{n}}(j)+N(j)\right|<3 A, 1<j<d\right\}+\sum_{j=1}^{d} P\{|N(j)|>A\} \\
& <P\left\{\left|S_{\sigma_{n}}(j)+N(j)\right|<3 A, 1<j<d\right\}+K_{3} \exp -\frac{A^{2}}{2 A^{2} k^{-1}} \\
& <P\left\{\left|S_{\sigma_{n}}(j)+N(j)\right|<3 A, 1<j<d\right\}+K_{3} \exp -\frac{k}{2} .
\end{aligned}
$$

Similarly

$$
\begin{aligned}
P\left\{\left|\sum_{i=1}^{n} x_{i}(j)+N(j)\right|<3 A, 1<j \leqslant d\right\} \\
<P\left\{\left|\sum_{1}^{n} x_{i}\right| \leqslant 4 d^{1 / 2} A\right\}+K_{3} \exp -\frac{k}{2} .
\end{aligned}
$$

If $\varphi_{1}, \varphi_{2}, \psi$ are the characteristic functions of, respectively, $G_{1}, G_{2}$ and $H$, then the characteristic functions of $S_{o_{n}}+N$ and $\Sigma_{1}^{n} \chi_{i}+N$ are

$$
\exp \left(-\frac{A^{2}}{2 k}|\theta|^{2}\right) \varphi_{2}(\theta)^{n} \psi(\theta)^{n} \text { and } \exp \left(-\frac{A^{2}}{2 k}|\theta|^{2}\right) \varphi_{1}(\theta)^{n} \psi(\theta)^{n}
$$

Thus, by the inversion formula,

$$
\begin{aligned}
& \mid P\left\{\left|S_{o_{n}}(j)+N(j)\right| \leqslant 3 A, 1 \leqslant j \leqslant d\right\} \\
& \quad-P\left\{\left|\sum_{i=1}^{n} x_{i}(j)+N(j)\right|<3 A, 1 \leqslant j \leqslant d\right\} \mid \\
& <\pi^{-d} \int_{-\infty}^{+\infty} \cdots \int_{-\infty}^{+\infty} d \theta_{1} \cdots d \theta_{d} \prod_{j=1}^{d}\left|\frac{\sin 3 \theta_{j} A}{\theta_{j}}\right| \\
& \quad \cdot\left|\varphi_{2}(\theta)^{n}-\varphi_{1}(\theta)^{n}\right| \exp \left(-\frac{A^{2}}{2 k}|\theta|^{2}\right)|\psi(\theta)|^{n} \\
& <\left(\frac{3 A}{\pi}\right)^{d} n \int_{-\infty}^{+\infty} \cdots \int_{-\infty}^{+\infty}\left|\varphi_{1}(\theta)-\varphi_{2}(\theta)\right| \exp \left(-\frac{A^{2}}{2 k}|\theta|^{2}\right) d \theta_{1} \cdots d \theta_{d} .
\end{aligned}
$$

But, by virtue of (2.8), 


$$
\begin{aligned}
\mid \varphi_{1}(\theta) & -\varphi_{2}(\theta)|=| \int e^{i\langle\theta, x\rangle} d\left(G_{1}(x)-G_{2}(x)\right) \mid \\
& =\left|\int\left\{e^{i\langle\theta, x\rangle}-\sum_{r=0}^{15} \frac{i^{r}\langle\theta, x\rangle^{r}}{r !}\right\} d\left(G_{1}(x)-G_{2}(x)\right)\right| \\
& <\frac{1}{16 !} \int\langle\theta, x\rangle^{16} d\left(G_{1}(x)+G_{2}(x)\right)=\frac{2}{16 !} \int\langle\theta, x\rangle^{16} d G_{2}(x) \\
& <\frac{2}{16 !}|\theta|^{16} \sum_{r=1}^{\infty} p_{0}^{r-1}\left(1-p_{0}\right)((r-1) L)^{16}
\end{aligned}
$$

(see (2.7) and recall that $G$ is concentrated on $C_{1}$ ). Consequently, for $A>$ $2^{k / 8}$ and $n<2^{k+2}$,

$$
\begin{aligned}
& \mid P\left\{\left|S_{o_{n}}(j)+N(j)\right|<3 A, 1<j<d\right\} \\
&-P\left\{\left|\sum_{i=1}^{n} x_{i}(j)+N(j)\right|<3 A, 1<j<d\right\} \mid \\
&< \Gamma_{6}(F) n A^{d} \int \cdots \int|\theta|^{16} \exp \left(-\frac{A^{2}}{2 k}|\theta|^{2}\right) d \theta_{1} \cdots d \theta_{d} \\
&<\Gamma_{7}(F) n A^{d-d-16} k^{(d+16) / 2}<\Gamma_{7}(F) k^{(d+16) / 2} 2^{-k+2} .
\end{aligned}
$$

Combined with (2.9) and (2.10) this yields

$$
P\left\{\left|S_{o_{n}}\right| \leqslant 2 A\right\} \leqslant P\left\{\left|\sum_{1}^{n} \chi_{i}\right| \leqslant 4 d^{1 / 2} A\right\}+\Gamma_{8}(F) e^{-k / 2} .
$$

Interchanging the subscripts 1 and 2 we prove similarly

$$
P\left\{\left|S_{o_{n}}\right|<A\right\}>P\left\{\left|\sum_{1}^{n} \chi_{i}\right| \leqslant \frac{1}{2} d^{-1 / 2} A\right\}-\Gamma_{8}(F) e^{-k / 2},
$$

for all $A \geqslant 2^{k / 8}, n \leqslant 2^{k+2}$. Finally, to prove our claim we appeal to the proof of Theorem 3 in [9]. Just as in the estimates on pp. 1179-1181 of [9],

$$
\begin{aligned}
P\left\{\left|S_{\sigma_{n}}\right|\right. & \left.<A \text { for some } 2^{k}<n<2^{k+1}\right\} \\
& <\left(\sum_{n=0}^{2^{k}} P\left\{\left|S_{\sigma_{n}}\right| \leqslant A\right\}\right)^{-1} \sum_{n=2^{k}}^{2^{k+2}-1} P\left\{\left|S_{\sigma_{n}}\right|<2 A\right\},
\end{aligned}
$$

and also 


$$
\begin{aligned}
& P\left\{\left|\sum_{1}^{n} x_{i}\right| \leqslant 4 d^{1 / 2} A \text { for some } 2^{k}<n<2^{k+2}\right\} \\
& \geqslant\left[\sum_{n=0}^{2^{k+2}} P\left\{\left|\sum_{1}^{n} \chi_{i}\right| \leqslant 8 d^{1 / 2} A\right\}\right]-1_{n=2^{k}}^{2^{k+2}-1} P\left\{\left|\sum_{1}^{n} \chi_{i}\right| \leqslant 4 d^{1 / 2} A\right\} \\
& >K_{4}\left[\sum_{n=0}^{2^{k}} P\left\{\left|\sum_{1}^{n} \chi_{i}\right|<\frac{1}{2} d^{-1 / 2} A\right\} \sum_{n=2^{k}}^{-1} \sum_{1}^{2^{k+2}-1} P\left\{\left|\sum_{1}^{n} \chi_{i}\right| \leqslant 4 d^{1 / 2} A\right\} .\right.
\end{aligned}
$$

Since

$$
\sum_{n=0}^{2^{k}} P\left\{\left|S_{\sigma_{n}}\right| \leqslant A\right\} \rightarrow \infty \text { as } k \rightarrow \infty \text { and } A \geqslant 2^{k / 8},
$$

it follows from (2.11)-(2.14) that for $k \geqslant k_{1}(F)$,

$$
\begin{aligned}
P\left\{\left|S_{\sigma_{n}}\right|<A \text { for some } 2^{k}<n<2^{k+1}\right\} \\
<2 K_{4}^{-1}\left\{P\left\{\left|\sum_{1}^{n} \chi_{i}\right|<4 d^{1 / 2} A \text { for some } 2^{k}<n<2^{k+1}\right\}\right. \\
\left.+P\left\{\left|\sum_{1}^{n} \chi_{i}\right|<4 d^{1 / 2} A \text { for some } 2^{k+1}<n<2^{k+2}\right\}\right) \\
+\Gamma_{9}(F) \exp (-k / 2) .
\end{aligned}
$$

Since $\Sigma_{1}^{n} \chi_{i}$ can be taken for $S_{n}\left(F_{1}\right)$ (its increments $\chi_{n}$ have distribution $F_{1}$ ), we see from this that if Proposition 1 holds for $S_{n}\left(F_{1}\right)$, then it also holds for $S_{n}\left(F_{2}\right)$ and for the original $S_{n}$. (Note that we can always obtain (1.4) for $k<k_{1}$ by increasing $\Gamma_{1}$.)

To complete the proof of the lemma we show finally that there exists a $G_{1}$ with a continuous density and satisfying (2.8). Let there be $M$ positive integer $d$-vectors $\nu$ with $1<\|\nu\| \leqslant 16$ and consider the set

$$
\mathfrak{N}=\left\{\left(z_{\nu}\right)_{1<\|\nu\|<16}:\left|z_{\nu}\right|<\Delta_{\nu}+1, z_{\nu}=\int x^{\nu} d R\right. \text { for some }
$$

$$
\text { distribution function } \left.R \text { on } \mathbf{R}^{d} \text { and all } 1<\|\nu\|<16\right\} \text {, }
$$

where the constants $\Delta_{\nu}<\infty$ are chosen large enough such that

$$
\mu_{\nu} \equiv \int x^{\nu} d G_{2}(x) \in\left[-\Delta_{\nu}, \Delta_{\nu}\right], \quad 1<\|\nu\|<16 .
$$

Then $\mathscr{R}$ is a bounded convex subset of $\mathbf{R}^{M}$ and $\mu=\left(\mu_{\nu}\right)_{1<\|v\|<16} \in \mathfrak{R}$. 
Assume first that $\mu \in \partial \mathscr{N}$. Then there exists a supporting hyperplane of $\Re$ at $\mu$ (see [3, Theorem 8D), i.e., there exist constants $c_{0}, c_{\nu}, 1 \leqslant\|\nu\| \leqslant 16$, not all zero, such that

$$
\sum_{1<\|\nu\|<16} c_{\nu} z_{\nu}+c_{0} \geqslant 0 \text { for all } z \in \Re
$$

and

$$
\sum_{1<\|\nu\|<16} c_{\nu} \mu_{\nu}+c_{0}=0
$$

Then, if

$$
P(x) \equiv \sum_{\nu=1}^{M} c_{\nu} x^{\nu}+c_{0}
$$

(2.15) implies that $\int P(x) d R(x) \geqslant 0$ for all distribution functions $R$ on $\mathbf{R}^{d}$ with

$$
\left|\int x^{\nu} d R(x)\right| \leqslant \Delta_{\nu}+1, \quad 1 \leqslant\|\nu\| \leqslant 16 .
$$

It is not hard to see from this and (2.16) that $P(x)=0$ for $x \in \operatorname{supp}\left(G_{2}\right)$, and, hence, for $x \in \operatorname{supp}\left(G^{* 0}\right)=\{0\}$ as well as for

$x \in \operatorname{supp}\left(G^{*(s)}\right)=\left\{x_{1}+\cdots+x_{s}: x_{i} \in \operatorname{supp} G\right\} \quad$ for some $s \geqslant 1$.

$P(0)=0$ shows that $c_{0}=0$. Moreover, if $y_{1}, \ldots, y_{d+1}$ are $(d+1)$ points in $\operatorname{supp}(G)=\operatorname{supp}(F) \cap C_{1}$ which do not lie in any hyperplane, then also, for any integers $k_{1} \geqslant 0, \ldots, k_{d+1} \geqslant 0, \Sigma k_{i} y_{i} \in \operatorname{supp}\left(G_{2}\right)$ and hence

$$
\sum_{1<\|\nu\|<16} c_{\nu}\left(k_{1}+\cdots+k_{d+1}\right)^{-16}\left(k_{1} y_{1}+\cdots+k_{d+1} y_{d+1}\right)^{\nu}=0 .
$$

If we let $k_{i} \rightarrow \infty$ such that

$$
k_{i}\left(k_{1}+\cdots+k_{d+1}\right)^{-1} \rightarrow \lambda_{i} \geqslant 0 \text { with } \sum_{i=1}^{d+1} \lambda_{i}=1,
$$

we obtain

$$
\sum_{\|\nu\|=16} c_{\nu}\left(\lambda_{1} y_{1}+\cdots+\lambda_{d+1} y_{d+1}\right)^{\nu}=0
$$

for all $\lambda_{i}>0$ with $\sum_{i=1}^{d+1} \lambda_{i}=1$, i.e.,

$$
\sum_{\|\nu\|=16} c_{\nu} x^{\nu}=0
$$

for all $x$ in the closed convex hull of $y_{1}, \ldots, y_{d+1}$. Since these $(d+1)$ points do not lie in any hyperplane, their convex hull has a nonempty interior (see [3, Theorem 4]) and we conclude $c_{\nu}=0$ for $\|\nu\|=16$. But then also 


$$
\sum_{1<\|\nu\|<15} c_{\nu}\left(k_{1}+\cdots+k_{d+1}\right)^{-15}\left(k_{1} y_{1}+\cdots+k_{d+1} y_{d+1}\right)^{\nu}=0
$$

and continuing in this way we find that all $c_{\nu}=0$. This was excluded, so that $\mu \in \partial \mathscr{N}$ is impossible. But then $\mu$ lies in the interior of $\mathscr{N}$ and for each of the $2^{M}$ choices of $\alpha_{\nu}$ with $\alpha_{\nu}=+1$ or -1 , we can find a point $z(\alpha) \in \Re$ such that

$$
\operatorname{sgn}\left(z_{\nu}(\alpha)-\mu_{\nu}\right)=\operatorname{sgn} \alpha_{\nu}
$$

and

$$
z_{\nu}(\alpha)=\int x^{\nu} d R_{\alpha}(x)
$$

for some $R_{\alpha}$ with a continuous density. Indeed any point of $\mathfrak{N}$ can be approximated by the moments of continuous densities on $\mathbf{R}^{d}$. It is a question of simple algebra only to deduce from (2.17) that some convex combination of the $z(\alpha)$ equals $\mu$. Say

$$
\mu_{\nu}=\sum_{\alpha} \gamma(\alpha) z_{\nu}(\alpha), \quad 1 \leqslant\|\nu\| \leqslant 16,
$$

with $\gamma(\alpha) \geqslant 0, \Sigma_{\alpha} \gamma(\alpha)=1$. Then clearly $G_{1} \equiv \Sigma_{\alpha} \gamma(\alpha) R_{\alpha}$ has the moments $\mu_{\nu}$ for $\|\nu\| \leqslant 16$ and has a continuous density as required.

From now on we assume that (2.1) holds. We introduce the following quantities: $A_{k}$ will be any fixed positive number not less than $2^{k / 8}$. $\omega$ will stand for a generic unit vector in $\mathbf{R}^{d}$ and for any such vector we set

$$
t(\omega)=t(\omega, k)=\min \left\{n: P\left\{\left|\left\langle S_{n}, \omega\right\rangle\right|>A_{k}\right\}>(8 d+8)^{-1}\right\} .
$$

As we shall see in the next lemma $t(\omega)$ is bounded on $|\omega|=1$, and we can therefore pick an $\omega_{d}=\omega_{d}^{k}$ which maximizes $t(\cdot, k)$, i.e. for which

$$
t\left(\omega_{d}^{k}\right)=\max _{|\omega|=1} t(\omega, k) \text {. }
$$

After that we can successively pick $\omega_{d-1}^{k}, \ldots, \omega_{1}^{k}$ such that $\left\langle\omega_{i}^{k}, \omega_{j}^{k}\right\rangle=\delta_{i, j}$ and

$$
t\left(\omega_{d-l-1}^{k}\right)=\max \left\{t(\omega, k):\left\langle\omega, \omega_{j}^{k}\right\rangle=0, d-l \leqslant j<d\right\} .
$$

We define

$$
T=T(k)=t\left(\omega_{2}^{k}, k\right)
$$

and note that by our construction $\omega_{1}, \ldots, \omega_{d}$ form an orthonormal basis for $\mathbf{R}^{d}$,

and if

$$
T(k) \leqslant t\left(\omega_{j}^{k}, k\right), \quad j \geqslant 3
$$

$$
\mathcal{H}=\mathcal{H}^{k}=\operatorname{span} \text { of }\left\{\omega_{1}^{k}, \omega_{2}^{k}\right\}=\left\{z: z=\alpha \omega_{1}^{k}+\beta \omega_{2}^{k}\right\}
$$


then

$$
t(\omega, k) \leqslant T(k) \text { for } \omega \in \mathcal{H} .
$$

Next we take $M\left(\omega_{2}^{k}, k\right)=1$ and for all $\omega \neq \omega_{2}$ we take $M(\omega)=M(\omega, k)$ as the maximum over $1<n<T(k)$ of any $1-(8 d+8)^{-1}$ quantile of $A_{k}^{-1}\left|\left\langle S_{n}, \omega\right\rangle\right|$, i.e.,

$$
P\left\{A_{k}^{-1}\left|\left\langle S_{n}, \omega\right\rangle\right|<M(\omega)\right\} \geqslant 1-(8 d+8)^{-1}, \quad 1<n<T(k),
$$

and for some $1<N(\omega)=N(\omega, k) \leqslant T(k)$,

$$
P\left\{A_{k}^{-1}\left|\left\langle S_{N}, \omega\right\rangle\right| \geqslant M(\omega)\right\} \geqslant(8 d+8)^{-1} \text {. }
$$

By definition of $T,(2.22)$ also holds for $\omega=\omega_{2}$ and $N\left(\omega_{2}, k\right)=T(k)$. Also, because $M(\omega, k)$ must be at least as big as a $1-(8 d+8)^{-1}$ quantile of $A_{k}^{-1}\left\langle S_{t(\omega, k)}, \omega\right\rangle$ if $t(\omega, k) \leqslant T$, we may and shall choose $M(\omega, k)$ such that

$$
M(\omega, k) \geqslant 1 \text { for } \omega \in \mathcal{H} \text {. }
$$

Observe also that $t\left(\omega_{j}\right) \geqslant T(k)$ for $j \geqslant 2$ and therefore

$$
\begin{aligned}
P\left\{\left|\left\langle S_{n}, \omega\right\rangle\right| \leqslant 2 A_{k}\right\} & \geqslant P\left\{\left|\left\langle S_{n-1}, \omega\right\rangle\right| \leqslant A_{k}\right\} P\left\{\left|X_{n}\right| \leqslant A_{k}\right\} \\
& \geqslant 1-(4 d+4)^{-1}
\end{aligned}
$$

whenever

$$
k \geqslant k_{2}(F), \quad n \leqslant T(k) \text { and } \omega=\omega_{j} \text { with } 2<j \leqslant d
$$

for a suitable $k_{2}(F)<\infty$.

LEMMA 2. There exists $a \Gamma_{10}=\Gamma_{10}(F)<\infty$ such that for all $|\omega|=1, k>1$,

$$
t(\omega, k)<\Gamma_{10} A_{k}^{2} \text {. }
$$

Moreover there exist a universal $K_{0}<\infty$ and $a k_{3}=k_{3}(F)<\infty$ such that for all $|\omega|=1, k \geqslant k_{3}$,

$$
T(k) P\left\{\left|\left\langle X_{1}, \omega\right\rangle\right|>M(\omega, k) A_{k}\right\}<K_{0}
$$

and $^{7}$

$$
\begin{gathered}
T(k) \sigma^{2}\left\{\left\langle X_{1}, \omega\right\rangle I\left[\left|\left\langle X_{1}, \omega\right\rangle\right|<M(\omega, k) A_{k}\right]\right\}<K_{0}\left\{M(\omega, k) A_{k}\right\}^{2} \\
T(k) \rightarrow \infty \text { and } A_{k} \inf _{|\omega|=1} M(\omega, k) \rightarrow \infty \text { as } k \rightarrow \infty
\end{gathered}
$$

and there exists a universal $0<\eta<1$ (see (2.44)) such that for all $|\omega|=1$, $k>1$,

$$
P\left\{\left|\left\langle S_{T}, \omega\right\rangle\right| \geqslant \frac{1}{2} M(\omega, k) A_{k}\right\}>\eta
$$

\footnotetext{
${ }^{7}$ For a random variable $Y, \sigma^{2}\{Y\}$ denotes its variance. $I[]$ denotes the indicator function of the event between square brackets.
} 
Proof. For any real random variable $Y$ its concentration function $Q(Y, \cdot)$ is defined by

$$
Q(Y, L)=\sup _{y} P\{y \leqslant Y<y+L\}
$$

It is easy to see and well known (see [7, Chapters 1.1 and 2.11) that this sup is taken on for some $y$, that for $0<L_{1}<L_{2}$,

$$
Q\left(Y, L_{2}\right)<\left(L_{1}^{-1} L_{2}+1\right) Q\left(Y, L_{1}\right)
$$

and that for $Y_{1}$ and $Y_{2}$ independent,

$$
Q\left(Y_{1}+Y_{2}, L\right)<\min \left\{Q\left(Y_{1}, L\right), Q\left(Y_{2}, L\right)\right\} .
$$

If $Y_{1}, Y_{2}, \ldots$ are independent and identically distributed with distribution function $G$, then Theorem 3.1 of Esseen [6] gives

$$
Q\left(\sum_{1}^{n} Y_{i}, L\right)<K_{5} n^{-1 / 2}\left\{L^{-2} \int_{|x|<2 L} x^{2} d G^{s}(x)+\int_{|x|>2 L} d G^{s}(x)\right\}^{-1 / 2}
$$

for some universal constant $K_{5}<\infty$, independent of $G, n$ and $L$. We shall now apply this with $Y_{i}=\left\langle X_{i}, \omega\right\rangle$ for an arbitrary unit vector $\omega$. First choose $L$ again such that $\operatorname{supp}(F) \cap C_{1}$ is not contained in any hyperplane, where $C_{1}$ is as in (2.2). Then

$$
\Gamma_{11}^{2} \equiv L^{-2} \inf _{|\omega|=1} \int_{|\langle x, \omega\rangle|<2 L}\langle x, \omega\rangle^{2} d F^{s}(x)>0,
$$

and thus by (2.31) and (2.33),

$$
\begin{aligned}
P\left\{\left|\left\langle S_{n}, \omega\right\rangle\right|<A_{k}\right\} & <2\left\{L^{-1} A_{k}+1\right\} Q\left(\left\langle S_{n}, \omega\right\rangle, L\right) \\
& <2\left\{L^{-1} A_{k}+1\right\} K_{5} \Gamma_{11}^{-1} n^{-1 / 2}<1-(8 d+8)^{-1}
\end{aligned}
$$

as soon as

$$
n>\left(\frac{8 d+8}{8 d+7}\right)^{2}\left\{2 L^{-1} A_{k}+2\right\}^{2} K_{5}^{2} \Gamma_{11}^{-2} .
$$

This implies (2.26). The same argument shows for any $L^{\prime}>L$,

$$
\begin{aligned}
P\left\{\left|\left\langle S_{n}, \omega\right\rangle\right|\left\langle\frac{1}{2} L^{\prime}\right\}\right. & <Q\left(\left\langle S_{n}, \omega\right\rangle, L^{\prime}\right) \\
& <\left(L^{-1} L^{\prime}+1\right) K_{5} \Gamma_{11}^{-1} n^{-1 / 2}<1-(8 d+8)^{-1}
\end{aligned}
$$

as soon as $n>\left(2 K_{5}\right)^{2}\left(L^{-1} L^{\prime}+1\right)^{2} \Gamma_{11}^{-2}$. Thus also $M(\omega) A_{k}>\frac{1}{2} L^{\prime}$ whenever $T(k)>\left(2 K_{5}\right)^{2}\left(L^{-1} L^{\prime}+1\right)^{2} \Gamma_{11}^{-2}$. However, we must have $T(k) \rightarrow \infty$ as $k \rightarrow$ $\infty$ because for each fixed $t$,

$$
\sup _{\omega} P\left\{\left|\left\langle S_{t}, \omega\right\rangle\right|>A_{k}\right\}<P\left\{\left|S_{t}\right| \geqslant 2^{k / 8}\right\} \rightarrow 0, \quad k \rightarrow \infty .
$$

Thus also for each fixed $L^{\prime}, A_{k} \inf _{\omega} M(\omega, k)>\frac{1}{2} L^{\prime}$ eventually, and (2.29) holds.

We turn to (2.27) and (2.28). We have for each $\omega$, 


$$
P\left\{\left|\left\langle S_{T-1}, \omega\right\rangle\right| \leqslant M(\omega) A_{k}\right\} \geqslant 1-(8 d+8)^{-1} \geqslant \frac{1}{2}
$$

(see (2.21) for $\omega \neq \omega_{2}$, and the definition of $T(k)$ and $M\left(\omega_{2}, k\right)=1$ for $\left.\omega=\omega_{2}\right)$. On the other hand, by (2.31) with $L_{1}=\frac{1}{8} M(\omega) A_{k}$ and (2.33), the left-hand side of (2.34) is bounded above by

$$
17 K_{5}(T-1)^{-1 / 2} P\left\{\left|\left\langle X_{1}^{s}, \omega\right\rangle\right|>\frac{1}{4} M(\omega) A_{k}\right\}^{-1 / 2},
$$

so that

$$
(T-1) P\left\{\left|\left\langle X_{1}^{s}, \omega\right\rangle\right|>\frac{1}{4} M(\omega) A_{k}\right\}<2^{12} K_{5}^{2} .
$$

Now (2.32) for $Y_{1}=Y, Y_{2}=Y^{\prime}$ shows that

$$
Q\left(Y^{s}, L\right) \leqslant Q(Y, L)
$$

and this, together with (2.35), yields

$$
\begin{aligned}
Q\left(\left\langle X_{1}, \omega\right\rangle, \frac{1}{2} M(\omega) A_{k}\right) \geqslant Q\left(\left\langle X_{1}^{s}, \omega\right\rangle, \frac{1}{2} M(\omega) A_{k}\right) \\
\quad \geqslant P\left\{\left|\left\langle X_{1}^{s}, \omega\right\rangle\right|<\frac{1}{4} M(\omega) A_{k}\right\} \geqslant 1-(T-1)^{-1} 2^{12} K_{5}^{2} .
\end{aligned}
$$

Finally, take $\Gamma_{12}=\Gamma_{12}(F)$ so large that

$$
P\left\{\left|X_{1}\right|>\Gamma_{12}\right\}<\frac{1}{2},
$$

and $k_{3}=k_{3}(F)$ so large that for all $k \geqslant k_{3}$,

$$
\frac{1}{2} A_{k} \inf _{\omega} M(\omega, k)>\Gamma_{12}
$$

as well as

$$
(T(k)-1)^{-1} 2^{12} K_{5}^{2}<\frac{1}{4} \quad \text { and } T(k)>2 .
$$

Then (2.36) shows that there exists some interval $\left[a, a+\frac{1}{2} M(\omega) A_{k}\right]$ which contains $\left\langle X_{1}, \omega\right\rangle$ with a probability at least $1-(T(k)-1)^{-1} 2^{12} K_{5}^{2} \geqslant \frac{3}{4}$. By (2.37), for $k \geqslant k_{3}$ this interval must intersect $\left[-\Gamma_{12},+\Gamma_{12}\right] \subset$ $\left[-\frac{1}{2} M(\omega) A_{k}, \frac{1}{2} M(\omega) A_{k}\right]$. Thus we proved for $k>k_{3}$,

$$
\begin{aligned}
P\left\{\left|\left\langle X_{1}, \omega\right\rangle\right| \leqslant M(\omega) A_{k}\right\} & \geqslant P\left\{\left\langle X_{1}, \omega\right\rangle \in\left[a, a+\frac{1}{2} M(\omega) A_{k}\right]\right\} \\
& \geqslant 1-(T(k)-1)^{-1} 2^{12} K_{5}^{2} .
\end{aligned}
$$

This proves $(2.27)$ for $k \geqslant k_{3}(F)$.

To obtain (2.28) we again apply (2.33) with $Y_{i}=\left\langle X_{i}, \omega\right\rangle$ and take $L=$ $M(\omega) A_{k}$. Combined with (2.31) and (2.34) this gives

$$
\begin{gathered}
3 K_{5}(T-1)^{-1 / 2} M(\omega) A_{k}\left\{\int_{|\langle x, \omega\rangle|<2 M(\omega) A_{k}}\langle x, \omega\rangle^{2} d F^{s}(x)\right\}^{-1 / 2} \\
>1-(8 d+8)^{-1}>\frac{1}{2},
\end{gathered}
$$

and therefore, 


$$
T(k) \int_{|\langle x, \omega\rangle|<2 M(\omega) A_{k}}\langle x, \omega\rangle^{2} d F^{s}(x)<2^{7}\left\{K_{5} M(\omega) A_{k}\right\}^{2} .
$$

Now let $Y$ be any random variable and $c \geqslant 0$ a constant. Denote the distribution function of $Y$ by $G$ and put

$$
d=\left\{\int_{|y|<c} d G(y)\right\}^{-1} \int_{|y|<c} y d G(y)=E\{Y|| Y \mid<c\} .
$$

Then $|d|<c$ and

$$
\begin{aligned}
& \int_{|y|<2 c} y^{2} d G^{s}(y) \geqslant \iint_{\substack{\left|y_{1}\right|<c \\
\left|y_{2}\right|<c}}\left(y_{1}-y_{2}\right)^{2} d G\left(y_{1}\right) d G\left(y_{2}\right) \\
& =\iint_{\substack{\left|y_{1}\right|<c \\
\left|y_{2}\right|<c}}\left\{\left(y_{1}-d\right)^{2}+2\left(y_{1}-d\right)\left(y_{2}-d\right)+\left(y_{2}-d\right)^{2}\right\} d G\left(y_{1}\right) d G\left(y_{2}\right) \\
& =2 \int_{\left|y_{2}\right|<c} d G\left(y_{2}\right) \int_{\left|y_{1}\right|<c}\left(y_{1}-d\right)^{2} d G\left(y_{1}\right) .
\end{aligned}
$$

Consequently,

$$
\begin{aligned}
& \sigma^{2}\{Y I[|Y|<c]\}<E\left\{(Y I[|Y|<c]-d)^{2}\right\} \\
& \quad=\int_{|y|<c}(y-d)^{2} d G(y)+d^{2} \int_{|y|>c} d G(y) \\
&<\frac{1}{2}(P\{|Y|<c\})^{-1} \int_{|y|<2 c} y^{2} d G^{s}(y)+P\{|Y|>c\} c^{2} .
\end{aligned}
$$

When this is applied to $Y=\left\langle X_{1}, \omega\right\rangle, c=M(\omega) A_{k}$, we obtain from the above inequality:

$$
\begin{aligned}
T(k) \sigma^{2}\left\{\left\langle X_{1}, \omega\right\rangle I\left[\left|\left\langle X_{1}, \omega\right\rangle\right| \leqslant M(\omega) A_{k}\right]\right\} \\
\leqslant\left(2^{6} K_{5}^{2}\left(P\left\{\left|\left\langle X_{1}, \omega\right\rangle\right| \leqslant M(\omega) A_{k}\right\}\right)^{-1}\right. \\
\left.+T(k) P\left\{\left|\left\langle X_{1}, \omega\right\rangle\right|>M(\omega) A_{k}\right\}\right)\left\{M(\omega) A_{k}\right\}^{2} .
\end{aligned}
$$

Together with (2.27)and (2.29) this implies (2.28).

Lastly we prove (2.30). For $\omega=\omega_{2}(2.30)$ is immediate since we already observed that (2.22) holds for $\omega=\omega_{2}$ with $N$ replaced by $T$. We therefore fix $\omega \neq \omega_{2}$ for the remainder of the proof. Since, for any $n\left\langle T,\left\langle S_{T}, \omega\right\rangle\right.$ is the sum of the independent random variables $\left\langle S_{\left[T_{n}{ }^{-1}\right] n}, \omega\right\rangle$ and $\left\langle S_{T-\left[T^{-1}\right] n}, \omega\right\rangle$, we have 


$$
\begin{aligned}
& P\left\{\left|\left\langle S_{T}, \omega\right\rangle\right|<\frac{1}{2} M(\omega) A_{k}\right\} \\
& <33 Q\left(\left\langle S_{T}, \omega\right\rangle, \frac{1}{32} M(\omega) A_{k}\right) \quad(\text { by }(2.31)) \\
& <33 Q\left(\left\langle S_{\left[T n^{-1}\right] n}, \omega\right\rangle, \frac{1}{32} M(\omega) A_{k}\right) \quad(\text { by }(2.32)) \\
& <33 K_{5}[T / n]^{-1 / 2}\left(P\left\{\left|\left\langle S_{n}^{s}, \omega\right\rangle\right|>\frac{1}{16} M(\omega) A_{k}\right\}\right)^{-1 / 2} \quad(\text { by }(2.33)) \\
& <33 K_{5}[T / n]^{-1 / 2}\left\{1-Q\left(\left\langle S_{n}, \omega\right\rangle, \frac{1}{8} M(\omega) A_{k}\right)\right\}^{-1 / 2} \quad \text { (as in (2.36)). }
\end{aligned}
$$

Now in order to prove (2.30) we only have to consider those $\omega$ for which

$$
P\left\{\left|\left\langle S_{T}, \omega\right\rangle\right| \geqslant \frac{1}{2} M(\omega) A_{k}\right\} \leqslant \frac{1}{2} .
$$

For such an $\omega$ (2.38) implies for all $n<T$,

$$
Q\left(\left\langle S_{n}, \omega\right\rangle, \frac{1}{8} M(\omega) A_{k}\right) \geqslant 1-2^{14} K_{5}^{2} n T^{-1} .
$$

Now apply (2.39) with $n=N^{\prime}$, where

$$
N^{\prime}=N^{\prime}(\omega, k)=\min \left\{n: P\left\{\left|\left\langle S_{n}, \omega\right\rangle\right| \geqslant \frac{1}{2} M(\omega) A_{k}\right\} \geqslant(8 d+8)^{-1}\right\} .
$$

Then, by $(2.22), N^{\prime}(\omega, k) \leqslant N(\omega, k) \leqslant T(k)$. Also, by the very definition of $N^{\prime}$,

$$
P\left\{\left\langle S_{N^{\prime}}, \omega\right\rangle>\frac{1}{2} M(\omega) A_{k}\right\}>(16)^{-1}(d+1)^{-1}
$$

or the inequality holds with $\left\langle S_{N^{\prime}}, \omega\right\rangle$ replaced by $-\left\langle S_{N^{\prime}}, \omega\right\rangle$. For the sake of definiteness assume (2.40). Then any interval containing $\left\langle S_{N^{\prime}}, \omega\right\rangle$ with a probability larger than $(16 d+15)(16 d+16)^{-1}$ must contain points to the right of $\frac{1}{2} M(\omega) A_{k}$. By (2.39) there exists such an interval of length $\frac{1}{8} M(\omega) A_{k}$ which contains $\left\langle S_{N^{\prime}}, \omega\right\rangle$ with a probability at least

$$
1-2{ }^{14} K_{5}^{2} N^{\prime} T^{-1}>(16 d+15) /(16 d+16)
$$

whenever $T / N^{\prime}>2{ }^{18} K_{5}^{2}(d+1)+4$. In this case, therefore,

$$
P\left\{\frac{3}{8} M(\omega) A_{k}<\left\langle S_{N^{\prime}}, \omega\right\rangle\right\}>1-2^{14} K_{5}^{2} N^{\prime} T^{-1}
$$

and

$$
\begin{aligned}
& P\left\{\left\langle S_{T}, \omega\right\rangle>\frac{1}{2} M(\omega) A_{k}\right\} \\
& >P\left\{\left\langle S_{(j+1) N^{\prime}}-S_{j N^{\prime}}, \omega\right\rangle>\frac{3}{8} M(\omega) A_{k},\right. \\
& \left.0<j<\left[\frac{T}{N^{\prime}}\right],\left\langle S_{T-\left[T\left(N^{\prime}\right)^{-1}\right] N^{\prime}, \omega}\right\rangle>-\frac{1}{2} M(\omega) A_{k}\right\} \\
& >\left(1-2^{14} K_{5}^{2} \frac{N^{\prime}}{T}\right)^{T / N^{\prime}} \min _{l<N^{\prime}} P\left\{\left|\left\langle S_{l}, \omega\right\rangle\right|<\frac{1}{2} M(\omega) A_{k}\right\} \\
& >\frac{8 d+7}{8 d+8} \exp -2^{15} K_{5}^{2} \quad \text { (use the definition of } N^{\prime} \text { ). }
\end{aligned}
$$


If $2<T / N^{\prime}<2{ }^{18} K_{5}^{2}(d+1)+4$, we use (2.40) instead of (2.41) and obtain, as in (2.42),

$$
\begin{aligned}
& P\left\{\left\langle S_{T}, \omega\right\rangle \geqslant \frac{1}{2} M(\omega) A_{k}\right\} \\
& >P\left\{\left\langle S_{(j+1) N^{\prime}}-S_{j N^{\prime}}, \omega\right\rangle \geqslant \frac{1}{2} M(\omega) A_{k},\right. \\
& \quad 0 \leqslant j<\left[\frac{T}{N^{\prime}}\right],\left\langle S_{\left.\left.T-\left[T\left(N^{\prime}\right)^{-1}\right]_{N^{\prime}}, \omega\right\rangle \geqslant \frac{1}{2} M(\omega) A_{k}\right\}}\right. \\
& >\left((16 d+16)^{-1}\right)^{T / N^{\prime}}(8 d+7)(8 d+8)^{-1} \\
& >(8 d+7)(8 d+8)^{-1} \exp -\left(2^{18} K_{5}^{2}(d+1)+4\right) \log (16 d+16) .
\end{aligned}
$$

Lastly, if $1 \leqslant T\left(N^{\prime}\right)^{-1}<2$, then $N^{\prime} \leqslant N \leqslant T<2 N^{\prime}$ and a fortiori $T-N$ $<N^{\prime}$. But then

$$
\begin{aligned}
& P\left\{\left|\left\langle S_{T}, \omega\right\rangle\right| \geqslant \frac{1}{2} M(\omega) A_{k}\right\} \\
&>P\left\{\left|\left\langle S_{N}, \omega\right\rangle\right| \geqslant M(\omega) A_{k}\right\} P\left\{\left|\left\langle S_{T-N}, \omega\right\rangle\right| \leqslant \frac{1}{2} M(\omega) A_{k}\right\} \\
& \geqslant \frac{1}{8 d+8} \min _{l<N^{\prime}} P\left\{\left|\left\langle S_{l}, \omega\right\rangle\right| \leqslant \frac{1}{2} M(\omega) A_{k}\right\} \quad(\text { by }(2.22)) \\
&\left.>(8 d+7)(8 d+8)^{-2} \quad \text { (by definition of } N^{\prime}\right) .
\end{aligned}
$$

Thus in all cases (2.30) holds with

$$
\eta=\frac{8 d+7}{8 d+8} \exp -\left(2^{18} K_{5}^{2}(d+1)+4\right) \log (16 d+16) .
$$

We can now give the reduction of Proposition 1 to a two dimensional problem. For the remainder of the proof we shall use the abbreviation

$$
m(k)=M\left(\omega_{1}^{k}, k\right) .
$$

Next we define

$$
\begin{aligned}
& J_{n}=J_{n}^{k}=I\left[\left|\left\langle X_{n}, \omega_{1}^{k}\right\rangle\right|>m(k) A_{k} \text { or }\left|\left\langle X_{n}, \omega_{2}^{k}\right\rangle\right|>A_{k}\right], \\
& \nu_{r}=\nu_{r}^{k}=\inf \left\{n: \sum_{l=1}^{n}\left(1-J_{l}\right)=r T(k)\right\}, \\
& Y_{r}=Y_{r}^{k}=\sum_{\nu_{r-1}<n<\nu_{r}} X_{n} .
\end{aligned}
$$

$Y_{r}$ is the sum over a bunch of $X_{n}$ 's, exactly $T(k)$ of which satisfy $\left|\left\langle X, \omega_{1}^{k}\right\rangle\right|<$ $m(k) A_{k}$ and $\left|\left\langle X, \omega_{2}^{k}\right\rangle\right|\left\langle A_{k}\right.$. It is easy to see from this that the $\left.Y_{r}, r\right\rangle 1$, are independent and identically distributed. Moreover, we can write

$$
Y_{r}=\sum_{\nu_{r-1}<n<\nu_{r}} X_{n} J_{n}+\sum_{\nu_{r-1}<n<\nu_{r}} X_{n}\left(1-J_{n}\right),
$$


and by definition the second term contains exactly $T(k)$ summands with $1-J_{n} \neq 0$. Thus if $\alpha_{1}, \alpha_{2}, \ldots, \beta_{1}, \beta_{2}, \ldots$ are independent, each $\alpha_{i}\left(\beta_{i}\right)$ with the conditional distribution of $X_{1}$, given $J_{1}=1$ (respectively $J_{1}=0$ ), then

$$
\sum_{v_{r-1}<n<\nu_{r}} X_{n}\left(1-J_{n}\right)
$$

has the same distribution as

$$
\sum_{n=1}^{T(k)} \beta_{n}
$$

Moreover, the sum (2.47) is independent of $\Sigma_{v_{r-1}<n<p_{r}} X_{n} J_{n}$, which has the same distribution as

$$
\sum_{n=1}^{\Lambda} \alpha_{n}
$$

where $\Lambda=\Lambda(k)$ is independent of all $\alpha_{i}, \beta_{i}$ and has the negative binomial distribution

$$
\begin{array}{rl}
P\{\Lambda=l\} & =P\left\{\nu_{r}-\nu_{r-1}=T(k)+l\right\} \\
& =\left(\begin{array}{c}
T(k)-1+l \\
l
\end{array}\right)\left(P\left\{J_{1}=1\right\}\right)^{l}\left(1-P\left\{J_{1}=1\right\}\right)^{T(k)} \\
l & l>0 .
\end{array}
$$

LEMma 3. There exist constants $\Gamma_{13}(F)-\Gamma_{15}(F)<\infty$ such that for $2^{k / 8}<$ $A_{k} \leqslant 2^{k / 2}$ and any choice of the unit vector $\omega_{0}=\omega_{0}^{k} \in \mathcal{H}^{k}$ and $k>1$ one has $P\left\{\left|S_{n}\right|<A_{k}\right.$ for some $\left.2^{k}<n<2^{k+1}\right\}<\Gamma_{13}\left\{A_{k} 2^{-k / 2}\right\}^{d-2}$

$$
\begin{gathered}
\sum_{2^{k-1}<r T(k)<\left(K_{0}+\Gamma_{10}+2\right) 2^{k}} P\left\{\left|\sum_{n=1}^{r} Y_{n}^{k}, \omega_{j}^{k}\right\rangle \mid\right. \\
\left.<4 M\left(\omega_{j}^{k}, k\right) A_{k} \text { for } 0 \leqslant j<2\right\} \\
+\Gamma_{14} \exp -\Gamma_{15} k .
\end{gathered}
$$

Proof. Let $\mathcal{S}_{n}$ be the $\sigma$-field generated by $X_{1}, \ldots, X_{n}$, and consider for some fixed $k$ the $\left\{\mathcal{G}_{n}\right\}$ stopping times

$$
\begin{aligned}
& \tau=\tau(k)=\min \left\{n: 2^{k}<n<2^{k+1},\left|S_{n}\right|<A_{k}\right\} \\
& \quad(=\infty \text { if no such } n \text { exists }), \\
& \sigma=\sigma(k)=\inf \left\{\nu_{r}: \nu_{r} \geqslant \tau\right\} .
\end{aligned}
$$


Then the set

$$
\begin{aligned}
& \left\{\tau<\infty, J_{l}=0 \text { for exactly } s \text { indices } l\right. \text { with } \\
& \left.\qquad l<\tau \text { but } l \text { greater than the last } \nu_{r}<\tau\right\}
\end{aligned}
$$

belongs to $\mathcal{G}_{\tau}$. Now fix an $\omega \in \mathcal{K} \cup\left\{\omega_{3}, \ldots, \omega_{d}\right\}$ and set

$$
M^{*}(\omega, k)= \begin{cases}M(\omega, k) & \text { if } \omega \in \mathcal{H}, \\ 1 & \text { if } \omega \in\left\{\omega_{3}, \ldots, \omega_{d}\right\} .\end{cases}
$$

Then $M^{*}(\omega, k) \geqslant 1$ by (2.23), and on the set (2.52),

$$
\begin{aligned}
P\left\{\left|\left\langle S_{\sigma}, \omega\right\rangle\right|>4 M^{*}(\omega, k) A_{k} \mid \mathcal{G}_{\tau}\right\} \\
\quad<P\left\{\left|\left\langle S_{\sigma}-S_{\tau}, \omega\right\rangle\right|>3 M^{*}(\omega, k) A_{k} \mid \mathcal{G}_{\tau}\right\} \\
\quad=P\left\{\left|\left\langle S_{\gamma}, \omega\right\rangle\right|>3 M^{*}(\omega, k) A_{k}\right\},
\end{aligned}
$$

where

$$
\gamma=\gamma(s, k)=\inf \left\{n: \sum_{i=1}^{n}\left(1-J_{i}\right)=T(k)-s\right\}
$$

(note that only $0<s<T(k)$ can occur).

By definition $\gamma(s, k) \geqslant T(k)-s$, and for each $L$ and $k>k_{2}(F)$,

$$
\begin{aligned}
& P\left\{\left|\left\langle S_{\gamma}, \omega\right\rangle\right|>3 M^{*}(\omega, k) A_{k}\right\} \\
&< P\left\{\left|\left\langle S_{T(k)-s}, \omega\right\rangle\right|>2 M^{*}(\omega, k) A_{k}\right\} \\
&+P\left\{\left|\left\langle S_{\gamma}-S_{T(k)-s}, \omega\right\rangle\right|>M^{*}(\omega, k) A_{k}\right\} \\
&<(4 d+4)^{-1}+P\{\gamma-(T(k)-s)>L\} \\
&+P\left\{\max _{j<L}\left|\left\langle S_{T(k)-s+j}-S_{T(k)-s}, \omega\right\rangle\right|>M^{*}(\omega, k) A_{k}\right\}
\end{aligned}
$$

(see (2.21) and (2.24)).

Now for all $s<T(k)$,

$$
\begin{gathered}
P\{\gamma(s)-(T(k)-s)>L\}=P\left\{\sum_{1}^{T(k)-s+L}\left(1-J_{i}\right)<T(k)-s\right\} \\
<P\left\{\sum_{1}^{T(k)+L} J_{i}>L\right\}<\frac{T(k)+L}{L} P\left\{J_{i}=1\right\} .
\end{gathered}
$$

Moreover, by (2.46) and (2.27), for $k>k_{3}(F)$,

$$
\begin{aligned}
P\left\{J_{i}^{k}=1\right\} & <P\left\{\left|\left\langle X_{1}, \omega_{i}^{k}\right\rangle\right|>M\left(\omega_{i}^{k}, k\right) A_{k} \text { for } i=1 \text { or } i=2\right\} \\
& <2 K_{0} T(k)^{-1} .
\end{aligned}
$$


Since $T(k) \rightarrow \infty$ with $k$ (see (2.29)) we can fix an $L$, independent of $s<T(k)$, such that for all $k \geqslant k_{3}$,

$$
P\{\gamma(s)-(T(k)-s)>L\}<2 K_{0}(T(k)+L)(L T(k))^{-1}<(8 d+8)^{-1} \text {. }
$$

With $L$ fixed in this way

$$
\begin{array}{r}
P\left\{\max _{j<L}\left|\left\langle S_{T(k)-s+j}-S_{T(k)-s}, \omega\right\rangle\right|>M^{*}(\omega, k) A_{k}\right\} \\
\quad<\sum_{j=0}^{L} P\left\{\left|S_{j}\right|>\inf _{|\omega|=1} M^{*}(\omega, k) A_{k}\right\}<\frac{1}{8 d+8}
\end{array}
$$

as soon as $\inf _{\omega} M^{*}(\omega, k) A_{k}$ is large enough. Thus by (2.29) we can find $k_{4}(F)<\infty$, such that for $k>k_{4}(F)$ one has on the set (2.52),

$$
P\left\{\left|\left\langle S_{\sigma}, \omega\right\rangle\right|>4 M^{*}(\omega, k) A_{k} \mid \mathcal{G}_{\tau}\right\}<(2 d+2)^{-1},
$$

uniformly in $\omega$ and $s<T(k)$. Now with $\omega_{j}, 1<j<d$, as right after Lemma 1 and $\omega_{0} \in \mathcal{X}$ we have

$$
\begin{aligned}
P\left\{\tau<\infty,\left|\left\langle S_{\sigma}, \omega_{j}\right\rangle\right|>4 M^{*}(\omega, k) A_{k} \text { for some } 0<j<d\right\} \\
\quad<P\{\tau<\infty\} \sum_{j=0}^{d} P\left\{\left|\left\langle S_{\sigma}, \omega_{j}\right\rangle\right|>4 M^{*}(\omega, k) A_{k} \mid \tau<\infty\right\}<\frac{1}{2} P\{\tau<\infty\} .
\end{aligned}
$$

After taking complements with respect to $\{\tau<\infty\}$ and using the fact that $\sigma$ equals some $\nu_{r}$, we obtain

$$
\begin{gathered}
P\left\{\left|S_{n}\right| \leqslant A_{k} \text { for some } 2^{k}<n<2^{k+1}\right\}=P\{\tau<\infty\} \\
<2 P\left\{\tau<\infty,\left|\left\langle S_{\sigma}, \omega_{j}\right\rangle\right|<4 M^{*}\left(\omega_{j}, k\right) A_{k} \text { for all } 0<j<d\right\} \\
<2 P\left\{\exists \nu_{r} \in\left[2^{k},\left(K_{0}+\Gamma_{10}+2\right) 2^{k}\right)\right. \text { with } \\
\left.\qquad\left\langle S_{v_{r}}, \omega_{j}\right\rangle \mid<4 M^{*}\left(\omega_{j}, k\right) A_{k} \text { for all } 0<j<d\right\} \\
+2 P\left\{\tau<\infty, \sigma \geqslant\left(K_{0}+\Gamma_{10}+2\right) 2^{k}\right\} .
\end{gathered}
$$

The last term in (2.54) is easily seen to be exponentially small. Indeed, $\tau<2^{k+1}$ whenever $\tau<\infty$ so that

$$
\begin{aligned}
P\{\tau<\infty, \sigma & \left.>\left(K_{0}+\Gamma_{10}+2\right) 2^{k}\right\} \\
& <E\left\{P\left\{\sigma-\tau>\left(K_{0}+\Gamma_{10}\right) 2^{k} \mid \mathcal{G}_{\tau}\right\} ; \tau<\infty\right\},
\end{aligned}
$$

and on the set (2.52),

$$
\begin{aligned}
& P\left\{\sigma-\tau>\left(K_{0}+\Gamma_{10}\right) 2^{k} \mid \mathcal{G}_{\tau}\right\}=P\left\{\gamma(s, k)>\left(K_{0}+\Gamma_{10}\right) 2^{k}\right\} \\
& \quad<P\left\{\gamma(s, k)-(T(k)-s)>K_{0} 2^{k}\right\}=P\left\{\sum_{1}^{T(k)-s+K_{0} 2^{k}} J_{i}>K_{0} 2^{k}\right\}
\end{aligned}
$$


(recall $T(k)-s<T(k)<\Gamma_{10} A_{k}^{2}<\Gamma_{10} 2^{k}$ by $(2.26)$ and $A_{k}<2^{k / 2}$ ). Since $T(k)-s<\Gamma_{10} 2^{k}$ and $P\left\{J_{i}=1\right\} \rightarrow 0$ as $k \rightarrow \infty$ (see (2.53)), standard exponential estimates show that the last member of (2.55) has an upper bound of the form $\Gamma_{16}(F) \exp -\Gamma_{17}(F) 2^{k}$ uniformly in $s<T(k)$ (see [10, Chapter 9 , problems 12-16]).

The first term in the last member of (2.54) is more troublesome. Recall that $F$ is now assumed to satisfy (2.1). By taking $C_{0}$ smaller if necessary we may assume that

$$
C_{0}=\left\{z \in \mathbf{R}^{d}:\left|\left\langle z, \omega_{j}\right\rangle-c_{j}\right|<\lambda, 1<j<d\right\}
$$

for some $c_{j}$ and $\lambda>0$. Then we can write $F=a(2 \lambda)^{d} G_{3}+\left(1-a(2 \lambda)^{d}\right) H_{3}$, where $G_{3}$ is the uniform distribution on $C_{0}$ and $H_{3}$ is some other distribution function on $\mathbf{R}^{d}$. Correspondingly, we may assume that $X_{n}=E_{n} \theta_{n}+(1-$ $\left.E_{n}\right) \psi_{n}$ where the $E_{n}, \theta_{i}, \psi_{j}$ are independent,

$$
P\left\{E_{n}=1\right\}=1-P\left\{E_{n}=0\right\}=a(2 \lambda)^{d}, \quad n \geqslant 1,
$$

each $\theta_{i}$ has distribution $G_{3}$ and each $\psi_{j}$ has distribution $H_{3}$. Now we want to estimate for $3<j<d$ the conditional probability of

$$
\left|\left\langle S_{v_{r}}, \omega_{j}\right\rangle\right|<4 M^{*}\left(\omega_{j}, k\right) A_{k}=4 A_{k}
$$

given $\nu_{r}=s, E_{n}=1$ for $n=n_{1}, n_{2}, \ldots, n_{p}\left(1<n_{1}<\cdots<n_{p}<s\right)$, but not for any other $n<s$, and given the values of $X_{n}, n \notin\left\{n_{1}, \ldots, n_{p}\right\}$, as well as $\left\langle X_{n}, \omega_{j}\right\rangle$ for $0<j<2$ and $n \in\left\{n_{1}, \ldots, n_{p}\right\}$. Given all these data we know that for $j>3$,

$$
\left\langle S_{\nu,}, \omega_{j}\right\rangle=\left\langle S_{s}, \omega_{j}\right\rangle=\sum_{n \in\left\{n_{1}, \ldots, n_{p}\right\}}\left\langle\theta_{n}, \omega_{j}\right\rangle+D_{j},
$$

where

$$
D_{j}=\sum_{\substack{l \notin\left\{n_{1}, \ldots, n_{p}\right\} \\ 1<l<s}}\left\langle X_{l}, \omega_{j}\right\rangle
$$

is some known constant. Moreover, we know the values of $\left\langle\theta_{n}, \omega_{j}\right\rangle$ for $n \in\left\{n_{1}, \ldots, n_{p}\right\}$ and $0<j<2$. (Note that also the event $\left\{\nu_{r}=s\right\}$ is determined only by $J_{1}, \ldots, J_{s}$ and hence by $\left\langle X_{l}, \omega_{1}\right\rangle,\left\langle X_{l}, \omega_{2}\right\rangle, 1<l<s$.) However, $\theta_{n}$ has a uniform distribution on $C_{0}$, and therefore the conditional distribution of $\left\langle\theta_{n}, \omega_{3}\right\rangle, \ldots,\left\langle\theta_{n}, \omega_{d}\right\rangle$, given $\left\langle\theta_{n}, \omega_{1}\right\rangle=x_{1}$ and $\left\langle\theta_{n} \omega_{2}\right\rangle=x_{2}$, is the same for all $x_{1}, x_{2}$, to wit the uniform distribution on the $(d-2)$ dimensional cube

$$
C_{2}=\left\{x=(x(3), \ldots, x(d)):\left|x(i)-c_{i}\right|<\lambda, 3<i<d\right\} .
$$

This remains true even if we condition on $\left\langle\theta_{n}, \omega_{0}\right\rangle$ as well, because $\left\langle\theta_{n}, \omega_{0}\right\rangle$ is a function of $\left\langle\theta_{n}, \omega_{1}\right\rangle$ and $\left\langle\theta_{n}, w_{2}\right\rangle$ (since $\omega_{0}$ lies in $\mathcal{K}$, the plane spanned by $\omega_{1}$ 
and $\omega_{2}$ ). Thus, if $\delta_{1}, \delta_{2}, \ldots$ are independent random variables, each one uniformly distributed on $C_{2}$, then

$$
\begin{aligned}
& P\left\{\left|\left\langle S_{\nu_{r}}, \omega_{j}\right\rangle\right|<4 M^{*}\left(\omega_{j}, k\right) A_{k} \text { for } 0 \leqslant j<d \mid \nu_{r}=s,\right. \\
& \left.\sum_{n=1}^{\nu_{r}} E_{n}=p,\left|\left\langle S_{v_{r}}, \omega_{j}\right\rangle\right|<4 M^{*}\left(\omega_{j}, k\right) A_{k} \text { for } 0<j<2\right\} \\
& <\sup _{D_{j}} P\left\{\left|\sum_{l=1}^{p} \delta_{l}(j)+D_{j}\right| \leqslant 4 M^{*}\left(\omega_{j}, k\right) A_{k}=4 A_{k} \text { for } 3<j<d\right\} .
\end{aligned}
$$

Now the corollary to Theorem 6.2 of [6] applies to the independent identically distributed $(d-2)$ vectors $\left\{\delta_{l}\right\}_{l>1}$ whose distribution is not concentrated on any $(d-3)$ dimensional hyperplane and therefore the last member of (2.57) is bounded by

$$
\Gamma_{18}(F) p^{-(d-2) / 2} \prod_{j=3}^{d}\left\{4 \lambda^{-1} A_{k}+1\right\}<\Gamma_{19}(F)\left\{A_{k} p^{-1 / 2}\right\}^{d-2}
$$

It follows that for fixed $r$,

$$
\begin{aligned}
& P\left\{\nu_{r} \in\left[2^{k},\left(K_{0}+\Gamma_{10}+2\right) 2^{k}\right)\right. \\
& \text { and } \left.\left|\left\langle S_{v_{r}}, \omega_{j}\right\rangle\right|<4 M^{*}\left(\omega_{j}, k\right) A_{k} \text { for } 0 \leqslant j \leqslant d\right\} \\
& <P\left\{\sum_{n=1}^{2^{k}} E_{n}<\frac{1}{2} 2^{k} P\left\{E_{1}=1\right\}\right\} \\
& +\Gamma_{19}(F) A_{k}^{d-2}\left(2^{k-1} P\left\{E_{1}=1\right\}\right)^{-(d-2) / 2} \\
& \cdot P\left\{\nu_{r} \in\left[2^{k},\left(K_{0}+\Gamma_{10}+2\right) 2^{k}\right), \sum_{n=1}^{v_{r}} E_{n}>2^{k-1} P\left\{E_{1}=1\right\}\right. \\
& \left.\left.\quad K S_{v_{r}}, \omega_{j}\right\rangle \mid<4 M^{*}\left(\omega_{j}, k\right) A_{k}=4 M\left(\omega_{j}, k\right) A_{k} \text { for } 0<j<2\right\}
\end{aligned}
$$

Since $P\left\{E_{1}=1\right\}=a(2 \lambda)^{d}$ is independent of $k$, the first term in the righthand side of $(2.58)$ is at most $\Gamma_{20}(F) \exp -\Gamma_{21}(F) 2^{k}$ (see [10, Chapter 9, problems 12-16]) and we obtain 


$$
\begin{gathered}
P\left\{\exists \nu_{r} \in\left[2^{k},\left(K_{0}+\Gamma_{10}+2\right) 2^{k}\right) \text { with }\left|\left\langle S_{\nu_{r}}, \omega_{j}\right\rangle\right|\right. \\
\left.<4 M^{*}\left(\omega_{j}, k\right) A_{k} \text { for all } 0<j<d\right\} \\
<\sum_{2^{k-1}<r T(k)<\left(K_{0}+\Gamma_{10}+2\right) 2^{k}}\left[\left(\Gamma_{20} \exp -\Gamma_{21} 2^{k}\right)+\left(\Gamma_{22}(F)\left\{A_{k} 2^{-k / 2}\right\}^{d-2}\right)\right. \\
\left.\cdot P\left\{\left|\left\langle S_{\nu_{r}}, \omega_{j}\right\rangle\right|<4 M\left(\omega_{j}, k\right) A_{k} \text { for } 0<j<2\right\}\right] \\
+P\left\{\nu_{r} \in\left[2^{k},\left(K_{0}+\Gamma_{10}+2\right) 2^{k}\right)\right. \text { for some } \\
\left.r<2^{k-1}(T(k))^{-1} \text { or } r \geqslant\left(K_{0}+\Gamma_{10}+2\right) 2^{k}(T(k))^{-1}\right\} .
\end{gathered}
$$

Since $\nu_{r}$ increases with $r$ and $\nu_{r} \geqslant r T(k)$ (by definition of $\nu_{r}$ ) the last term in the right-hand side of (2.59) is at most

$$
P\left\{\nu_{n}>2^{k} \text { for } n=\left[2^{k-1}(T(k))^{-1}\right]\right\}
$$

and we leave it to the reader to show that this term again is bounded by $\Gamma_{23^{\circ}}$ $\exp -\Gamma_{24} 2^{k}$. Thus, since $S_{\nu_{r}}=\sum_{n=1}^{r} Y_{n}$, the last member of (2.54) is indeed bounded by the right-hand side of $(2.51)$ for $k \geqslant \max \left(k_{2}, k_{3}, k_{4}\right)$ and suitable $\Gamma_{13}-\Gamma_{15}$. Obviously we can then insure the validity of (2.51) for $k<$ $\max \left(k_{2}, k_{3}, k_{4}\right)$ by increasing $\Gamma_{14}$.

3. Completion of the proof. Lemma 3 shows that Proposition 1 will follow once we show that there exist $k_{5}(F)<\infty$ and $K_{2}<\infty$ such that for a suitable choice of $\omega_{0}^{k} \in \mathcal{H}^{k}$,

$$
\begin{aligned}
\sum_{r=s}^{2 s} P\left\{\left|\sum_{n=1}^{r}\left\langle Y_{n}^{k}, \omega_{j}^{k}\right\rangle\right|<4 M\left(\omega_{j}^{k}, k\right) A_{k} \text { for } 0<j<2\right\} \\
\leqslant K_{2}, \text { for all } k \geqslant k_{5}, s \geqslant 1 \text { and } A_{k} \in\left[2^{k / 8}, 2^{k / 2}\right] .
\end{aligned}
$$

Note that we can always obtain (1.4) for $k<k_{5}$ by increasing $\Gamma_{1}$; moreover, (1.4) is vacuously true for $A>2^{k / 2}$, and, as observed before, (1.4) follows from (2.6) when $A \leqslant 2^{k / 8}$. Note also that the last statement of our theorem will be immediate from Proposition 1 , because (1.2)-(1.4) imply for all $b \geqslant 0$,

$$
\begin{aligned}
& P\left\{\psi(n)^{-1}\left|S_{n}\right| \leqslant b \text { for some } n \geqslant 2^{k}\right\} \\
& <\sum_{l=k}^{\infty} P\left\{\left|S_{n}\right| \leqslant 2^{1 / 2} b \psi\left(2^{l}\right) \text { for some } 2^{l} \leqslant n<2^{l+1}\right\} \\
& <\Gamma_{1}(F)\left\{\sum_{l=k}^{\infty}\left\{2^{1 / 2} b \psi\left(2^{l}\right)+1\right\}^{d-2} 2^{-l(d-2) / 2}+\exp -l \Gamma_{2}(F)\right\} \\
& <K_{3} \Gamma_{1}(F) b^{d-2} \int_{2^{k-1}}^{\infty} \psi(t)^{d-2} t^{-d / 2} d t+K_{3} \Gamma_{1}(F) 2^{-k(d-2) / 2} \\
& \quad+\Gamma_{1}(F)\left\{1-\exp -\Gamma_{2}(F)\right\}^{-1} \exp -k \Gamma_{2}(F) \rightarrow 0 \quad(k \rightarrow \infty) .
\end{aligned}
$$


Moreover, it is well known that if (1.2) holds, then $\psi(n)^{-1}\left|S_{n}^{*}\right| \rightarrow \infty$ w.p.1 or $\psi(t)^{-1}|B(t)| \rightarrow \infty$ w.p.1 if and only if (1.3) holds (see [2, Theorems 5,6] or [8, \$4.2.15]; for $S_{n}^{*}$ one can also use the multidimensional analogue of Remark 2, p. 1182 in [9]). Thus to prove our theorem it suffices to prove (1.4), and this in turn has been reduced to proving (3.1).

This will be done by means of certain inequalities on two dimensional concentration functions which are contained in Proposition 2 and some corollaries. We therefore begin with their proofs; the notation is as in Proposition 2.

Proof of Proposition 2. By [6, formula (6.9)]

$$
\begin{aligned}
& \sup _{z \in \mathbb{R}^{2}} P\left\{\left|\sum_{1}^{n} Z_{i}+z\right| \leqslant \rho\right\} \\
& \left\langle K_{4} \rho^{2} \int_{|\theta|<\rho^{-1}} d \theta \exp -\left[\frac{1}{2} \sum_{i=1}^{n} \int_{\mathbf{R}^{2}}(1-\cos \langle\theta, u\rangle) d G_{i}^{s}(u)\right]\right. \\
& <K_{4} \rho^{2} \int_{|\theta|<\rho^{-1}} d \theta \exp -\left[\frac { 1 } { 2 } \sum _ { i = 1 } ^ { n } \left\{\int_{|u|<\rho_{i}}(1-\cos \langle\theta, u\rangle) d G_{i}^{s}(u)\right.\right. \\
& +\frac{1}{2 q_{i}} \int_{|u|,|v|>\rho_{i}}[(1-\cos \langle\theta, u\rangle) \\
& \left.\left.+(1-\cos \langle\theta, v\rangle)] d G_{i}^{s}(u) d G_{i}^{s}(v)\right\}\right] \\
& \left\langleK _ { 4 } \rho ^ { 2 } \int _ { | \theta | < \rho ^ { - 1 } } \operatorname { e x p } \left\{-K_{5} \sum_{i=1}^{n} \int_{|u|<\rho_{i}}\langle\theta, u\rangle^{2} d G_{i}^{s}(u)-\sum_{i=1}^{n} \frac{1}{4 q_{i}}\right.\right. \\
& \cdot \int_{u, v \in A_{i}}[(1-\cos \langle\theta, u\rangle)+(1-\cos \langle\theta, v\rangle)] \\
& \left.d G_{i}^{s}(u) d G_{i}^{s}(v)\right\} d \theta \text {. }
\end{aligned}
$$

Now take $C$ as in (1.7) and apply Hölder's inequality, as in [5, p. 212] or [6, p. 294]. It then follows that the last member of (3.2) is bounded by

$$
\begin{array}{r}
K_{4} \rho^{2}\left\{\int_{|\theta|<\rho^{-1}} d \theta \exp -K_{5} C|\theta|^{2}\right\}^{C^{-1} \Sigma_{i-1}^{n} \sigma_{i}^{2}} \cdot \exp \sum_{i} \frac{\rho_{i}^{2}}{C q_{i}} \int_{u, v \in A_{i}} d G_{i}^{s}(u) d G_{i}^{s}(v) \\
\cdot \log \int_{|\theta|<\rho^{-1}} d \theta \exp -\frac{C}{4 \rho_{i}^{2}}(2-\cos \langle\theta, u\rangle-\cos \langle\theta, v\rangle) .
\end{array}
$$


Clearly

$$
\int_{|\theta|<\rho^{-1}} d \theta \exp -K_{5} C|\theta|^{2} \leqslant \int_{\mathbf{R}^{2}} d \theta \exp -K_{5} C|\theta|^{2}=\frac{\pi}{K_{5} C} .
$$

It is also clear that

$$
\int_{|\theta|\left\langle\rho^{-1}\right.} d \theta \exp -\frac{C}{4 \rho_{i}^{2}}(2-\cos \langle\theta, u\rangle-\cos \langle\theta, v\rangle)
$$

is invariant under a rotation of the pair of vectors $u, v$ and therefore equals $\left(\varphi=\varphi(u, v)\right.$; recall also $|u|,|v|>\rho \geqslant \rho_{i}$ for $\left.u, v \in A_{i}\right)$

$$
\begin{aligned}
\int_{|\theta|<\rho^{-1}} d \theta \exp & -\frac{C}{4 \rho_{i}^{2}}\left[\left(1-\cos \theta_{i}|u|\right)\right. \\
& \left.+\left(1-\cos \left(\theta_{1}|v| \cos \varphi+\theta_{2}|v| \sin \varphi\right)\right)\right] \\
& <\int_{\left|\theta_{1}\right|<\rho^{-1}} d \theta_{1} \exp -\frac{C}{4 \rho_{i}^{2}}\left[1-\cos \theta_{1}|u|\right] \\
& \quad \sup _{b \in \mathrm{R}} \int_{\left|\theta_{2}\right|<\rho^{-1}} d \theta_{2} \exp -\frac{C}{4 \rho_{i}^{2}}\left[1-\cos \left(b+\theta_{2}|v| \sin \varphi\right)\right] \\
< & K_{6} \frac{\rho_{i}}{|u| C^{1 / 2}}\left(|u| \rho^{-1}+1\right) \cdot \frac{\rho_{i}}{|v||\sin \varphi| C^{1 / 2}}\left(|v||\sin \varphi| \rho^{-1}+1\right) \\
& \quad(\operatorname{compare}[5, \mathrm{p} .213]) \\
& \frac{2}{C} K_{6}\left(1+\frac{\rho_{i}}{|v||\sin \varphi(u, v)|}\right) .
\end{aligned}
$$

(1.8) now follows by putting these estimates together.

Corollary 1 is immediate from Proposition 1 by specialization (take all $\rho_{i}=\rho$ and $A_{i}=B_{1} \times B_{2}$ ). More important for our proof, though, is the more technical

Corollary 2. Let $Z_{1}, Z_{2}, \ldots$ be independent identically distributed two-vectors and assume that there exist constants $d_{1}>0,-\infty<d_{2}<\infty, d_{3}>0$ such that

$$
p_{1} \equiv P\left\{Z_{1}^{s}(2)>d_{1}, Z_{1}^{s}(1)<d_{2} Z_{1}^{s}(2)\right\}>0
$$

as well as

$$
p_{2} \equiv P\left\{Z_{1}^{s}(2)>d_{1}, Z_{1}^{s}(1) \geqslant d_{2} Z_{1}^{s}(2)+d_{3}\right\}>0 \text {. }
$$

Then for all $\rho \geqslant 0$ and all $n \geqslant 1$, 


$$
\sup _{z} P\left\{\left|\sum_{1}^{n} Z_{i}+z\right| \leqslant \rho\right\}<\Gamma_{3} \frac{1}{n}\left\{\left(\frac{\rho}{d_{1}}\right)^{2}+1\right\},
$$

for some constant $\Gamma_{3}=\Gamma_{3}\left(d_{i}, p_{i}\right)<\infty$ depending on $p_{1}, p_{2}, d_{1}-d_{3}$ only. In fact $\Gamma_{3}<\Gamma_{4}(D)<\infty$ for all $p_{i}, d_{i}$ and $D<\infty$ with

$$
\left|d_{2}\right|<D, \quad d_{1} d_{3}^{-1}<D, \quad p_{1} \geqslant D^{-1}, \quad p_{2} \geqslant D^{-1} \text {. }
$$

Moreover, for all $s>1$ and $\rho>0,8$

$$
E \#\left\{r \in[s, 2 s]:\left|\sum_{1}^{r} Z_{i}\right|<\rho\right\}<\Gamma_{3}\left\{\left(\frac{\rho}{d_{1}}\right)^{2}+1\right\}(1+\log 2) .
$$

If instead of (3.3) and (3.4) we assume that for some $d_{1}>0,-\infty<d_{2}<\infty$, and $d_{3}>0$,

$$
p_{3} \equiv P\left\{Z_{1}(2)>d_{1}, Z_{1}(1) \leqslant d_{2} Z_{1}(2)\right\}>0
$$

as well as

$$
p_{4} \equiv P\left\{Z_{1}(2)>d_{1}, Z_{1}(1) \geqslant d_{2} Z_{1}(2)+d_{3}\right\}>0,
$$

then for each $s \geqslant 1, \rho \geqslant 0$,

$$
E \#\left\{r \in[s, 2 s]:\left|\sum_{1}^{r} Z_{n}\right|<\rho\right\}<\Gamma_{5}\left\{\left(\frac{\rho}{d_{1}}\right)^{2}+1\right\},
$$

now with $\Gamma_{5}=\Gamma_{5}\left(p_{i}, d_{i}\right)<\infty$ depending only on $p_{3}, p_{4}$ and $d_{1}-d_{3}$. Moreover, $\Gamma_{5}<\Gamma_{6}(D)<\infty$ for all $p_{i}, d_{i}$ and $D<\infty$ with

$$
\left|d_{2}\right| \leqslant D, \quad d_{1} d_{3}^{-1} \leqslant D, \quad p_{3} \geqslant D^{-1}, \quad p_{4} \geqslant D^{-1} \text {. }
$$

Lastly, assume that (3.8) and (3.9) hold and that $W_{1}, W_{2}, \ldots$ are independent random vectors each with the distribution of $\sum_{i=1}^{\Lambda} Z_{i}$, where $\Lambda$ is independent of the $Z_{i}$, has the distribution given by (2.50), and that for some $d_{4}, d_{5}$,

$$
0<d_{4} \leqslant T(k) P\left\{J_{1}=1\right\} \leqslant d_{5}<\infty, \quad P\left\{J_{1}=1\right\} \leqslant \frac{1}{2} .
$$

Under these conditions one has for all $\rho \geqslant 0$,

$$
\sup _{z} P\left\{\left|\sum_{r=1}^{n} W_{r}+z\right| \leqslant \rho\right\} \leqslant \Gamma_{7} \frac{1}{n}\left\{\left(\frac{\rho}{d_{1}}\right)^{2}+1\right\}
$$

for some $\Gamma_{7}=\Gamma_{7}\left(p_{i}, d_{i}\right)$ depending on $p_{3}, p_{4}$ and $d_{1}-d_{5}$ only. Moreover, $\Gamma_{7}<$ $\Gamma_{8}(D)<\infty$ for all $p_{i}, d_{i}$ and $D<\infty$ which satisfy (3.11) and $d_{4} \geqslant D^{-1}$, $d_{5}<D$. Estimates (3.10) and (3.13) remain valid if (3.8) and (3.9) hold with $Z_{1}$ replaced by $-Z_{1}$ in one or both of these formulae.

Proof. We first prove (3.5) with $\rho=d_{1}$ from (3.3) and (3.4). This is an immediate application of Corollary 1 with

\footnotetext{
${ }^{8} \#\{\}$ denotes the number of elements in the set between braces.
} 


$$
B_{1}=\left\{z \in \mathbf{R}^{2}: z(2)>d_{1}, z(1)<d_{2} z(2)\right\}
$$

and

$$
B_{2}=\left\{z \in \mathbf{R}^{2}: z(2)>d_{1}, z(1) \geqslant d_{2} z(2)+d_{3}\right\} \quad \text { (see figure). }
$$

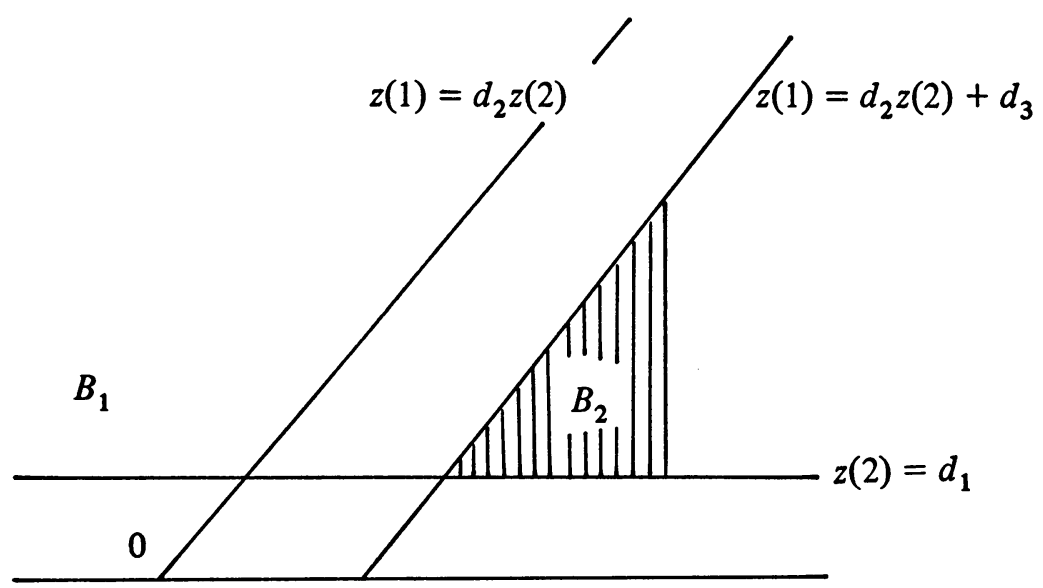

Clearly, $B_{1}, B_{2} \subset\left\{z \in \mathbf{R}^{2}:|z|>d_{1}\right\}$ and

$$
\int_{B_{i}} d G^{s}(u)=p_{i} \leqslant q=\int_{|u|>d_{1}} d G^{s}(u), \quad i=1,2 .
$$

Moreover, for $u \in B_{1}, v \in B_{2}$,

$$
|\sin \varphi(u, v)|=(|u||v|)^{-1}|u(2) v(1)-u(1) v(2)|,
$$

and, consequently,

$$
\begin{aligned}
|v||\sin \varphi(u, v)| & \geqslant \frac{u(2)}{|u(1)|+u(2)}\left|v(1)-\frac{u(1)}{u(2)} v(2)\right| \\
& \geqslant \frac{u(2)}{|u(1)|+u(2)}\left\{d_{2} v(2)+d_{3}-\frac{u(1)}{u(2)} v(2)\right\} .
\end{aligned}
$$

Now $u(1)<d_{2} u(2)$ for $u \in B_{1}$ so that the last member of (3.14) is positive. If $|u(1)|<\left(1+2\left|d_{2}\right|\right) u(2)$, then it is even bounded below by $\frac{1}{2}\left(1+\left|d_{2}\right|\right)^{-1} d_{3}$; and if $|u(1)|>\left(1+2\left|d_{2}\right|\right) u(2)$, then necessarily $u(1)<-2\left|d_{2}\right| u(2)$ and the last member of (3.14) is bounded below by

$$
\frac{u(2)}{|u(1)|+u(2)} \frac{1}{2} \frac{|u(1)|}{u(2)} v(2) \geqslant \frac{1}{4} \frac{1}{1+\left|d_{2}\right|} d_{1} .
$$

Thus in all cases

$$
|v||\sin \varphi(u, v)| \geqslant c \equiv \frac{1}{4} \frac{1}{1+\left|d_{2}\right|} \min \left\{d_{1}, d_{3}\right\}
$$


and (3.5) follows from (1.10) with

$$
\begin{aligned}
\Gamma_{3} & \equiv K_{1}\left(1+d_{1} c^{-1}\right) q\left(p_{1} p_{2}\right)^{-1} \\
& <K_{1}\left(p_{1} p_{2}\right)^{-1}\left\{1+4\left(1+\left|d_{2}\right|\right)\left(1+d_{1} d_{3}^{-1}\right)\right\} .
\end{aligned}
$$

Recall that we took $\rho=d_{1}$ so far. However, once we have (3.5) for $\rho=d_{1}$ it follows for each $\rho>0$ because any disc of radius $\rho$ can be covered by at most $K_{7}\left(\rho^{2} d_{1}^{-2}+1\right)$ discs of radius $d_{1}$. It is immediate from (3.16) that $\Gamma_{3}$ is bounded above whenever $p_{i}, d_{i}$ satisfy (3.6). Also (3.7) is immediate from (3.5) because $\sum_{r=s}^{2 s} r^{-1}<1+\log 2$.

Next we prove (3.13) by showing that (3.3) and (3.4) hold for suitable values of $p_{1}, p_{2}$ and $Z$ replaced by $W$. For this purpose take $B_{1}$ as above. Assume further that $z_{1}, z_{2}, z_{1}^{\prime} \in B_{1}$ satisfy

$$
z_{1}(1)-d_{2} z_{1}(2) \leqslant \min \left\{z_{2}(1)-d_{2} z_{2}(2), z_{1}^{\prime}(1)-d_{2} z_{1}^{\prime}(2)\right\}
$$

as well as

$$
z_{2}(2) \geqslant z_{1}^{\prime}(2)
$$

Then

$$
\begin{gathered}
z_{1}(1)+z_{2}(1)-z_{1}^{\prime}(1)-d_{2}\left(z_{1}(2)+z_{2}(2)-z_{1}^{\prime}(2)\right) \\
<z_{2}(1)-d_{2} z_{2}(2) \leqslant 0,
\end{gathered}
$$

and

$$
z_{1}(2)+z_{2}(2)-z_{1}^{\prime}(2)>z_{1}(2)>d_{1} \text {. }
$$

(3.19) and (3.20) just say that $z_{1}+z_{2}-z_{1}^{\prime} \in B_{1}$, so that $z_{1}+z_{2}-z_{1}^{\prime} \in B_{1}$ as soon as (3.17) and (3.18) hold. In agreement with the notational convention from before Lemma 1 we now take $\Lambda^{\prime}$ an independent copy of $\Lambda$ and put

$$
W_{1}=\sum_{i=1}^{\Lambda} Z_{i}, \quad W_{1}^{\prime}=\sum_{i=1}^{\Lambda^{\prime}} Z_{i}^{\prime}, \quad W_{1}^{s}=W_{1}-W_{1}^{\prime} .
$$

By comparing the different orders of the quantities in (3.17) and (3.18) we now obtain

$$
\begin{aligned}
P\left\{W_{1}^{s} \in B_{1}\right\} \geqslant & P\left\{\Lambda=2, \Lambda^{\prime}=1\right\} \\
& \cdot \int_{\substack{z_{1}, z_{2}, z_{1}^{\prime} \in B_{1} \\
(3.17) \text { and }(3.18) \text { hold }}} P\left\{Z_{1} \in d z_{1}, Z_{2} \in d z_{2}, Z_{1}^{\prime} \in d z_{1}^{\prime}\right\} \\
> & \frac{1}{6} P\{\Lambda=2\} P\left\{\Lambda^{\prime}=1\right\} \\
& \cdot \int_{z_{1}, z_{2}, z_{1}^{\prime} \in B_{1}} P\left\{Z_{1} \in d z_{1}\right\} P\left\{Z_{2} \in d z_{2}\right\} P\left\{Z_{1}^{\prime} \in d z_{1}^{\prime}\right\} \\
= & \frac{1}{6} P\{\Lambda=2\} P\left\{\Lambda^{\prime}=1\right\}\left(P\left\{Z_{1} \in B_{1}\right\}\right)^{3} .
\end{aligned}
$$


Now, by (2.50) and (3.12),

$$
P\{\Lambda=2\}>\frac{1}{2 !}\left(T(k) P\left\{J_{1}=1\right\}\right)^{2} \exp -2 d_{5}>\frac{1}{2} d_{4}^{2} \exp -2 d_{5} .
$$

Similarly,

$$
P\{\Lambda=1\} \geqslant d_{4} \exp -2 d_{5},
$$

and finally, $P\left\{Z_{1} \in B_{1}\right\}=p_{3}$ (by (3.8)). Thus

$$
\begin{aligned}
P\left\{W_{1}^{s}(2)\right. & \left.>d_{1}, W_{1}^{s}(1)<d_{2} W_{1}^{s}(2)\right\} \\
& =P\left\{W_{1}^{s} \in B_{1}\right\} \geqslant K_{8} d_{4}^{3} p_{3}^{3} \exp -4 d_{5} .
\end{aligned}
$$

Next consider $P\left\{W_{1}^{s} \in B_{2}\right\}$. Assume $z_{1}, z_{2}, z_{1}^{\prime} \in B_{2}$ are such that

$$
z_{1}(1)-d_{2} z_{1}(2) \geqslant \max \left\{z_{2}(1)-d_{2} z_{2}(2), z_{1}^{\prime}(1)-d_{2} z_{1}^{\prime}(2)\right\},
$$

and (3.18) holds; then we get

$$
\begin{aligned}
z_{1}(1)+z_{2}(1)-z_{1}^{\prime}(1) & -d_{2}\left(z_{1}(2)+z_{2}(2)-z_{1}^{\prime}(2)\right) \\
& \geqslant z_{2}(1)-d_{2} z_{2}(2) \geqslant d_{3},
\end{aligned}
$$

and as before, $z_{1}(2)+z_{2}(2)-z_{1}^{\prime}(2)>d_{1}$. Thus, as in (3.21) and (3.22), $P\left\{W_{1}^{s} \in B_{2}\right\} \geqslant P\left\{\Lambda=2, \Lambda^{\prime}=1\right\}$

$$
\begin{aligned}
& \cdot \int_{\substack{z_{1}, z_{2}, z_{1}^{\prime} \in B_{2} \\
(3.18) \text { and }(3.23) \text { hold }}} P\left\{Z_{1} \in d z_{1}, Z_{2} \in d z_{2}, Z_{1}^{\prime} \in d z_{1}^{\prime}\right\} \\
> & K_{8} d_{4}^{3} \exp -4 d_{5}\left(P\left\{Z_{1} \in B_{2}\right\}\right)^{3} \\
> & K_{8} d_{4}^{3} p_{4}^{3} \exp -4 d_{5} .
\end{aligned}
$$

(3.22) and (3.24) show that (3.3) and (3.4) hold for $W_{1}^{s}$ instead of $Z_{1}^{s}$ and

$$
p_{1}=K_{8} d_{4}^{3} p_{3}^{3} \exp -4 d_{5}, \quad p_{2}=K_{8} d_{4}^{3} p_{4}^{3} \exp -4 d_{5} \text {. }
$$

(3.13) is therefore immediate from (3.5). Also the bound $\Gamma_{7}<\Gamma_{8}(D)$, whenever (3.11) holds together with $d_{4}>D^{-1}, d_{5}<D$, is immediate from (3.25) and the fact that $\Gamma_{3}<\Gamma_{4}(D)$ on (3.6). Since we may everywhere in this argument replace $Z_{1}$ by $-Z_{1}$ without influencing the distribution of $W_{1}^{s}$, no change is needed if $Z_{1}$ is replaced by $-Z_{1}$ in (3.8) or (3.9).

Lastly, we prove (3.10). For this purpose let $\Lambda_{1}, \Lambda_{2}, \ldots$ be a sequence of independent random variables, also independent of the $Z_{i}$, and

$$
P\left\{\Lambda_{i}=l\right\}=\left(\frac{1}{2}\right)^{l+1}, \quad l>0 .
$$

(3.26) corresponds to $T(k)=1, P\left\{J_{1}=1\right\}=\frac{1}{2}$ in (2.50). Thus (3.12) is satisfied with $d_{4}=d_{5}=\frac{1}{2}$ in this case. Set $\tau_{0}=0$, and for $r>1$,

$$
\tau_{r}=\sum_{i=1}^{r} \Lambda_{i}, \quad W_{r}=\sum_{i=\tau_{r-1}+1}^{\tau_{r}} Z_{i} .
$$


Then the $W_{r}$ are independent, identically distributed and by what we just proved (3.13) holds with $\Gamma_{7}$ depending only on $p_{3}, p_{4}, d_{1}-d_{3}$ (since now $\left.d_{4}=d_{5}=\frac{1}{2}\right)$ and $\Gamma_{7}<\Gamma_{8}(D)$ whenever (3.11) holds. Moreover,

$$
\begin{gathered}
E \#\left\{r \in[s, 2 s]:\left|\sum_{1}^{r} Z_{i}\right|<\rho\right\}<(s+1) P\left\{\tau_{[s / 2]}>s \text { or } \tau_{4 s}<2 s\right\} \\
+E \#\left\{r \in\left[\tau_{[s / 2]}, \tau_{4 s}\right):\left|\sum_{1}^{r} Z_{i}\right|<\rho\right\}
\end{gathered}
$$

By (3.26),

$$
E \Lambda_{i}=1 \text {, }
$$

and by standard exponential bounds for the geometric distribution (compare $[9$, formulae (5.40)-(5.42)]),

$$
\begin{aligned}
P\left\{\left|\tau_{n}-n\right| \geqslant \frac{1}{2} n\right\} & =P\left\{\sum_{1}^{n} \Lambda_{i} \leqslant \frac{1}{2} n \text { or } \sum_{1}^{n} \Lambda_{i} \geqslant \frac{3}{2} n\right\} \\
& <K_{9} \exp -K_{10} n .
\end{aligned}
$$

Thus, the first term in the right-hand side of (3.28) is at most

$$
2(s+1) K_{9} \exp -K_{10} \frac{s}{2}<K_{11} \text {. }
$$

The second term in the right-hand side of (3.28) equals

$$
\begin{aligned}
& \sum_{[s / 2]<j<4 s} E \#\left\{r \in\left[\tau_{j}, \tau_{j+1}\right):\left|\sum_{i=1}^{r} Z_{i}\right|<\rho\right\} \\
& =\sum_{[s / 2]<j<4 s} \sum_{m=0}^{\infty} P\left\{\left|\sum_{1}^{\tau_{j}+m} Z_{i}\right|<\rho, \tau_{j+1}-\tau_{j}>m\right\} \\
& =\sum_{[s / 2]<j<4 s} \sum_{m=0}^{\infty} \int_{\mathbf{R}^{2}} P\left\{\tau_{j+1}-\tau_{j}>m, \sum_{l=\tau_{j}+1}^{\tau_{j}+m} Z_{l} \in d z\right\} \\
& \quad \cdot P\left\{\left|\sum_{i=1}^{\tau_{j}} Z_{i}+z\right|<\rho\right\} \\
& <\sum_{[s / 2]<j<4 s} \sum_{m=0}^{\infty} P\left\{\tau_{j+1}-\tau_{j}>m\right\} \sup _{2} P\left\{\left|\sum_{1}^{j} W_{i}+z\right|<\rho\right\} \\
& <\sum_{[s / 2]<j<4 s} E\left\{\tau_{j+1}-\tau_{j}\right\} \Gamma_{7} \frac{1}{j}\left\{\left(\frac{\rho}{d_{1}}\right)^{2}+1\right\} \text { (by (3.13)) } \\
& <\Gamma_{7} K_{12}\left\{\left(\frac{\rho}{d_{1}}\right)^{2}+1\right\} \quad(\text { by }(3.29)) .
\end{aligned}
$$


The last estimate works only for $s \geqslant 2$, but for $s=1(3.10)$ needs no proof anyway. From (3.28), (3.31) and the estimate for the first term in the right-hand side of (3.28), we finally see that (3.28) is, for $s>2$, at most

$$
K_{11}+\Gamma_{7} K_{12}\left\{\left(\frac{\rho}{d_{1}}\right)^{2}+1\right\} \leqslant K_{13}\left(\Gamma_{7}+1\right)\left\{\left(\frac{\rho}{d_{1}}\right)^{2}+1\right\} \text {. }
$$

We now return to the proof of the bound in (3.1). The notation will be as in $\S 2$ for the remainder of this section. We distinguish two cases:

$$
T(k) P\left\{J_{1}^{k}=1\right\}<2^{-5} \eta
$$

and

$$
2^{-5} \eta<T(k) P\left\{J_{1}^{k}=1\right\}<2 K_{0} .
$$

( $\eta$ and $K_{0}$ are as in Lemma 2. (3.33) and (3.34) are the only possibilities as we saw in (2.53).) For the remainder of the proof we are only interested in $\omega$ components with $\omega \in \mathcal{K}$, the plane spanned by $\omega_{1}, \omega_{2}$. We shall therefore think of all random variables as being two dimensional and specified by their $\omega_{1}$ and $\omega_{2}$ component. We remind the reader of the notation $m(k)=$ $M\left(\omega_{1}^{k}, k\right)$ and of the choice $M\left(\omega_{2}^{k}, k\right)=1$. For any random vector $X$ we define another random vector $\tilde{X} \in \mathcal{H C}$ by scaling of the $\omega_{1}$ and $\omega_{2}$ component as follows:

$$
\tilde{X}=\tilde{X}^{k}=A_{k}^{-1}\left\{\frac{1}{m(k)}\left\langle X, \omega_{1}^{k}\right\rangle \omega_{1}^{k}+\left\langle X, \omega_{2}^{k}\right\rangle \omega_{2}^{k}\right\} .
$$

For most quantities we shall no longer indicate its dependence on $k$ explicitly.

LEMmA 4. There exist $k_{6}=k_{6}(F)<\infty$ and $K_{14}<\infty$ such that the left-hand side of (3.1) is bounded by $K_{14}$ for all $s>1$ and all $k \geqslant k_{6}(F)$ for which (3.33) holds.

Proof. By dropping the condition on the $\omega_{0}$ component and using the above notation we see that the left-hand side of (3.1) is bounded above by

$$
\sum_{r=s}^{2 s} P\left\{\left|\sum_{n=1}^{r}\left\langle\tilde{Y}_{n}, \omega_{j}\right\rangle\right| \leqslant 4 \text { for } j=1,2\right\} \text {. }
$$

Of course,

$$
\begin{aligned}
\tilde{Y}_{r}= & \sum_{n=\nu_{r-1}+1}^{\nu_{r}}\left\{\left\langle\tilde{X}_{n}\left(1-J_{n}\right), \omega_{1}\right\rangle \omega_{1}+\left\langle\tilde{X}_{n}\left(1-J_{n}\right), \omega_{2}\right\rangle \omega_{2}\right\} \\
& +\sum_{n=\nu_{r-1}+1}^{\nu_{r}}\left\{\left\langle\tilde{X}_{n} J_{n}, \omega_{1}\right\rangle \omega_{1}+\left\langle\tilde{X}_{n} J_{n}, \omega_{2}\right\rangle \omega_{2}\right\} .
\end{aligned}
$$


By the observations made immediately following the introduction of $Y_{r}$ in $\$ 2$ (see (2.47)) the two sums in the right-hand side of (3.37) are independent, and if we call the first sum $\tilde{Z}_{r}$, i.e.,

$$
\tilde{Z}_{r}=\sum_{n=\nu_{r-1}+1}^{\nu_{r}}\left\{\left\langle\tilde{X}_{n}\left(1-J_{n}\right), \omega_{1}\right\rangle \omega_{1}+\left\langle\tilde{X}_{n}\left(1-J_{n}\right), \omega_{2}\right\rangle \omega_{2}\right\},
$$

then the two dimensional analogue of (2.32) gives

$$
\begin{aligned}
& \sup _{z} P\left\{\left|\sum_{n=1}^{r}\left\langle\tilde{Y}_{n}, \omega_{j}\right\rangle+z_{j}\right|<4, j=1,2\right\} \\
& \quad<\sup _{z} P\left\{\left|\sum_{n=1}^{r}\left\langle\tilde{Z}_{n}, \omega_{j}\right\rangle+z_{j}\right|<4, j=1,2\right\} .
\end{aligned}
$$

Moreover, as observed in (2.47) and (2.48), each $\tilde{Z}_{l}$ has the distribution of $\sum_{n=1}^{T(k)} \tilde{\beta}_{n}$, where the $\beta_{n}$ are independent, and each with the distribution of $X_{1}$, given $J_{1}=0$. Thus the variance of each $\left\langle\tilde{Z}_{l}, \omega\right\rangle$ equals

$$
\sigma^{2}(\omega, k) \equiv T(k) \sigma^{2}\left\{\left\langle\tilde{X}_{1}, \omega\right\rangle \mid J_{1}=0\right\} .
$$

Note also that

$$
\left|\tilde{X}_{1}\right|<A_{k}^{-1}\left(m_{k}^{-1}\left|\left\langle X_{1}, \omega_{1}\right\rangle\right|+\left|\left\langle X_{2}, \omega_{2}\right\rangle\right|\right)<2
$$

whenever $J_{1}=0$. Put

$$
\Delta_{k}=\inf \left\{\sigma^{2}(\omega, k):|\omega|=1, \omega \in \mathcal{K}\right\}
$$

and apply the corollary to Theorem 6.2 of Esseen [6] to the independent and identically distributed random two-vectors $\tilde{\beta}_{1}, \ldots, \tilde{\beta}_{r T(k)}$. Esseen's $\chi_{1}(4)$ can now be taken at least

$$
2 \inf _{|\omega|=1} \sigma^{2}\left\{\left\langle\tilde{\beta}_{1}, \omega\right\rangle\right\}=2 T(k)^{-1} \Delta_{k},
$$

and therefore the right-hand side of (3.38) is bounded by

$$
K_{15}\left(r T(k) T(k)^{-1} \Delta_{k}\right)^{-1}=K_{15}\left(r \Delta_{k}\right)^{-1} \text {. }
$$

Consequently, the left-hand side of (3.1) is at most

$$
\sum_{r=s}^{2 s} K_{15}\left(r \Delta_{k}\right)^{-1}<\frac{K_{15}}{\Delta_{k}}(1+\log 2) \text {. }
$$

Thus we have a bound of the desired form for (3.1) as soon as $\Delta_{k}$ exceeds some strictly positive $\varepsilon_{1}^{2}$. We choose $\varepsilon_{1}$ as follows:

$$
\begin{gathered}
K_{16}=32 K_{0}^{1 / 2} \eta^{-1 / 2}+4, \quad K_{17}=\frac{1}{16}\left(10 K_{0}^{1 / 2} \eta^{-1 / 2}+2\right)^{-1}, \\
\varepsilon_{1}=\frac{1}{32} \eta^{1 / 2} \frac{K_{17}^{2}}{K_{17}+8 K_{16}} .
\end{gathered}
$$


( $K_{0}$ and $\eta$ are as in Lemma 2.) For the remainder of the lemma we may now assume

$$
\Delta_{k}<\varepsilon_{1}^{2}
$$

By definition of $\Delta_{k}$ this means that there exists a unit vector $\Omega_{1} \in \mathcal{K}$ with $\sigma^{2}\left(\Omega_{1}, k\right)<2 \varepsilon_{1}^{2}$. By Chebyshev's inequality we then have, with $\mu=E\left\langle\tilde{Z}_{1}, \Omega_{1}\right\rangle$ and $T=T(k)$,

$$
\begin{aligned}
P\left\{\left|\left\langle\tilde{S}_{T}, \Omega_{1}\right\rangle-\mu\right| \geqslant 8 \varepsilon_{1} \eta^{-1 / 2}\right\} \\
\quad<P\left\{\tilde{S}_{T} \neq \tilde{Z}_{1}\right\}+P\left\{\left|\left\langle\tilde{Z}_{1}, \Omega_{1}\right\rangle-\mu\right|>8 \varepsilon_{1} \eta^{-1 / 2}\right\} \\
\quad<P\left\{J_{n}=1 \text { for some } n<T\right\}+\sigma^{2}\left(\Omega_{1}, k\right) / 64 \varepsilon_{1}^{2} \eta^{-1} \\
<\eta / 32+\eta / 32 \text { (by }(3.33) \text { and }(3.41))=\eta / 16 .
\end{aligned}
$$

We first show that (3.42) necessitates

$$
|\mu|>K_{17}-8 \varepsilon_{1} \eta^{-1 / 2}>\frac{1}{2} K_{17}
$$

for $k>k_{2}(F)+k_{3}(F)$. Indeed if (3.43) fails, then (3.42) shows

$$
P\left\{\left|\left\langle\tilde{S}_{T}, \Omega_{1}\right\rangle\right|>K_{17}\right\}<\eta / 2 \text {. }
$$

But if

and we define

$$
\Omega_{1}=\left(\cos \varphi_{0}\right) \omega_{1}+\left(\sin \varphi_{0}\right) \omega_{2}
$$

$$
\Omega_{2}=\left\{m(k)^{-2} \cos ^{2} \varphi_{0}+\sin ^{2} \varphi_{0}\right\}^{-1 / 2}\left(\frac{\cos \varphi_{0}}{m(k)} \omega_{1}+\left(\sin \varphi_{0}\right) \omega_{2}\right),
$$

then (3.44) says

$$
P\left\{\left|\left\langle S_{T}, \Omega_{2}\right\rangle\right|>K_{17}\left\{m(k)^{-2} \cos ^{2} \varphi_{0}+\sin ^{2} \varphi_{0}\right\}^{-1 / 2} A_{k}\right\}<\eta / 2 .
$$

At the same time $\Omega_{2}$ is a unit vector in $\mathcal{K}$, and thus by $(2.23), M\left(\Omega_{2}, k\right)>1$, and by (2.30),

$$
P\left\{\left|\left\langle S_{T}, \Omega_{2}\right\rangle\right|>\frac{1}{2} A_{k}\right\}>P\left\{\left|\left\langle S_{T}, \Omega_{2}\right\rangle\right|>\frac{1}{2} M\left(\Omega_{2}\right) A_{k}\right\}>\eta .
$$

(3.45) and (3.46) together imply

$$
K_{17}\left\{m(k)^{-2} \cos ^{2} \varphi_{0}+\sin ^{2} \varphi_{0}\right\}^{-1 / 2}>\frac{1}{2}
$$

and

$$
\sin ^{2} \varphi_{0}<4 K_{17}^{2}<\frac{3}{4}, \quad\left|\cos \varphi_{0}\right|>\frac{1}{2} .
$$

However, we also have from (2.30):

$$
P\left\{\left|\left\langle S_{T}, \omega_{1}\right\rangle\right|>\frac{1}{2} m(k) A_{k}\right\}>\eta ;
$$


and from (2.24):

$$
P\left\{\left|\left\langle S_{T}, \omega_{2}\right\rangle\right|<2 A_{k}\right\}>\frac{3}{4} \quad \text { for } k>k_{2}(F) .
$$

(3.44) and (3.48) together give

$$
\begin{aligned}
\frac{\eta}{2} & <P\left\{\left|\left\langle S_{T}, \omega_{1}\right\rangle\right| \geqslant \frac{1}{2} m(k) A_{k} \text { and }\left|\left\langle\tilde{S}_{T}, \Omega_{1}\right\rangle\right|<K_{17}\right\} \\
& =P\left\{\left|\left\langle S_{T}, \omega_{1}\right\rangle\right| \geqslant \frac{1}{2} m(k) A_{k}\right. \text { and } \\
& \left.\quad\left|\left\langle S_{T}, \omega_{1}\right\rangle \cos \varphi_{0}+\left\langle S_{T}, \omega_{2}\right\rangle \sin \varphi_{0} m(k)\right|<K_{17} m(k) A_{k}\right\} \\
& <P\left\{\left|\left\langle S_{T}, \omega_{2}\right\rangle \sin \varphi_{0} m(k)\right|>m(k) A_{k}\left(\frac{1}{2}\left|\cos \varphi_{0}\right|-K_{17}\right)>\frac{1}{8} m(k) A_{k}\right\} \\
& <P\left\{\left|\left\langle S_{T}, \omega_{2}\right\rangle\right| \geqslant\left(16 K_{17}\right)^{-1} A_{k}\right\} \quad \text { (by (3.47)). }
\end{aligned}
$$

(3.49) and (3.50) together show that any interval which contains $\left\langle S_{T}, \omega_{2}\right\rangle$ with a probability $\left(1-\frac{1}{4} \eta\right)$ must contain both $2 A_{k}$ and $\left(16 K_{17}\right)^{-1} A_{k}$ or both $-2 A_{k}$ and $-\left(16 K_{17}\right)^{-1} A_{k}$. Thus any such interval must have length at least

$$
\left\{\left(16 K_{17}\right)^{-1}-2\right\} A_{k} \geqslant 10 K_{0}^{1 / 2} \eta^{-1 / 2} A_{k} \quad \text { (see (3.39)). }
$$

For $k>k_{3}(F)$, however, this contradicts

$$
\begin{aligned}
P\left\{\left|\left\langle S_{T}, \omega_{2}\right\rangle-\sum_{n=1}^{T(k)} E\left\{\left\langle X_{n}, \omega_{2}\right\rangle I\left[\left|\left\langle X_{n}, \omega_{2}\right\rangle\right|<A_{k}\right]\right\}\right|>4 K_{0}^{1 / 2} \eta^{-1 / 2} A_{k}\right\} \\
<\sum_{n=1}^{T(k)} P\left\{\left|\left\langle X_{n}, \omega_{2}\right\rangle\right|>A_{k}\right\} \\
\quad+\frac{\eta}{16} \frac{T(k)}{K_{0} A_{k}^{2}} \sigma^{2}\left\{\left\langle X_{1}, \omega_{2}\right\rangle I\left[\left|\left\langle X_{n}, \omega_{2}\right\rangle\right|<A_{k}\right]\right\} \\
<T(k) P\left\{J_{1}=1\right\}+\frac{K_{0}}{K_{0}} \frac{\eta}{16} \quad\left(\text { by }(2.28) \text { and } M\left(\omega_{2}\right)=1\right) \\
<\eta / 8 \quad \text { (by (3.33)). }
\end{aligned}
$$

Thus, the assumption that (3.43) fails indeed leads to a contradiction when $k>k_{2}+k_{3}$, and for the remainder we may use (3.43) when $k>k_{6}(F) \equiv$ $k_{2}(F)+k_{3}(F)$.

Now bring in the unit vector

orthogonal to $\Omega_{1}$ in $\mathcal{X}$. If

$$
\Omega_{3}=\left(-\sin \varphi_{0}\right) \omega_{1}+\left(\cos \varphi_{0}\right) \omega_{2}
$$

$$
Q\left(\left\langle\tilde{S}_{T}, \Omega_{3}\right\rangle, K_{17}\right) \geqslant 1-\eta / 4,
$$


then there exists a constant $f$ such that

$$
P\left\{\left|\left\langle\tilde{S}_{T}, \Omega_{3}\right\rangle-f\right|<\frac{1}{2} K_{17}\right\} \geqslant 1-\eta / 4,
$$

which together with (3.42) and (3.40) would imply

$$
P\left\{\left|\tilde{S}_{T}-\mu \Omega_{1}-f \Omega_{3}\right| \leqslant \frac{1}{2} K_{17}+8 \varepsilon_{1} \eta^{-1 / 2}<K_{17}\right\}>1-\eta / 2,
$$

and a fortiori for $\Omega_{4}$ a unit vector in $\mathcal{H}$ orthogonal to $\mu \Omega_{1}+f \Omega_{3}$,

$$
P\left\{\left|\left\langle\tilde{S}_{T}, \Omega_{4}\right\rangle-\left\langle\mu \Omega_{1}+f \Omega_{3}, \Omega_{4}\right\rangle\right|=\left|\left\langle\tilde{S}_{T}, \Omega_{4}\right\rangle\right|<K_{17}\right\} \geqslant 1-\eta / 2 .
$$

Apart from the change from $\Omega_{1}$ to $\Omega_{4}$ this again is (3.44), which we showed to be impossible. Thus (3.52) must fail and no interval of length $K_{17}$ can contain $\left\langle\tilde{S}_{T}, \Omega_{3}\right\rangle$ with probability $1-\eta / 4$. Thus, if $\nu$ is a median of $\left\langle\tilde{S}_{T}, \Omega_{3}\right\rangle$, then

$$
P\left\{\left|\left\langle\tilde{S}_{T}, \Omega_{3}\right\rangle-\nu\right|>\frac{1}{2} K_{17}\right\} \geqslant \eta / 4
$$

and at least one of the inequalities

$$
P\left\{\left\langle\tilde{S}_{T}, \Omega_{3}\right\rangle>\nu+\frac{1}{2} K_{17}\right\} \geqslant \eta / 8
$$

or

$$
P\left\{\left\langle\tilde{S}_{T}, \Omega_{3}\right\rangle<\nu-\frac{1}{2} K_{17}\right\} \geqslant \eta / 8
$$

must hold. For the sake of definiteness assume (3.53) holds. Since $\nu$ is a median of $\left\langle\tilde{S}_{T}, \Omega_{3}\right\rangle$ we also have

$$
P\left\{\left\langle\tilde{S}_{T}, \Omega_{3}\right\rangle \leqslant \nu\right\} \geqslant \frac{1}{2} .
$$

We are almost ready to apply Corollary 2; we merely need an estimate on $\nu$, or, more generally, the distribution of $\left|\tilde{S}_{T}\right|$. Just as in (3.51) we have for any $x \geqslant 0$ and $k \geqslant k_{6}(F)$,

$$
\begin{aligned}
& P\left\{\left|\left\langle\tilde{S}_{T}, \omega_{1}\right\rangle-T(k) E\left\{\left\langle\tilde{X}_{1}, \omega_{1}\right\rangle I\left[\left|\left\langle X_{1}, \omega_{1}\right\rangle\right| \leqslant m(k) A_{k}\right]\right\}\right|>x\right\} \\
& <T(k) P\left\{\left|\left\langle X_{1}, \omega_{1}\right\rangle\right|>m(k) A_{k}\right\} \\
& \quad+\frac{T(k)}{x^{2}} \sigma^{2}\left\{\left\langle\tilde{X}_{1}, \omega_{1}\right\rangle I\left[\left|\left\langle X_{1}, \omega_{1}\right\rangle\right| \leqslant m(k) A_{k}\right]\right\} \\
& <T(k) P\left\{J_{1}=1\right\} \\
& \quad+\frac{T(k)}{x^{2}}\left\{m(k) A_{k}\right\}^{-2} \sigma^{2}\left\{\left\langle X_{1}, \omega_{1}\right\rangle I\left[\left|\left\langle X_{1}, \omega_{1}\right\rangle\right| \leqslant m(k) A_{k}\right]\right\} \\
& <\eta / 32+x^{-2} K_{0} \quad(\text { see }(3.33) \text { and }(2.28)) .
\end{aligned}
$$

But also by (2.21), 


$$
\begin{aligned}
P\left\{\left|\left\langle\tilde{S}_{T}, \omega_{1}\right\rangle\right|<2\right\} & =P\left\{\left|\left\langle S_{T}, \omega_{1}\right\rangle\right|<2 m(k) A_{k}\right\} \\
& \geqslant 1-(8 d+8)^{-1}>\frac{3}{4} .
\end{aligned}
$$

Together with (3.56) this shows (take $x=8 K_{0}^{1 / 2} \eta^{-1 / 2}$ in (3.56))

$$
\left|T(k) E\left\{\left\langle\tilde{X}_{1}, \omega_{1}\right\rangle \mid\left[\left|\left\langle X_{1}, \omega_{1}\right\rangle\right|<m(k) A_{k}\right]\right\}\right|<8 K_{0}^{1 / z} \eta^{-1 / 2}+2,
$$

and therefore (again take $x=8 K_{0}^{1 / 2} \eta^{-1 / 2}$ in (3.56))

$$
P\left\{\left|\left\langle\tilde{S}_{T}, \omega_{1}\right\rangle\right| \geqslant 16 K_{0}^{1 / 2} \eta^{-1 / 2}+2\right\}<\eta / 16 .
$$

The same inequality holds when $\omega_{1}$ is replaced by $\omega_{2}$ (use (3.49) to get (3.57)) and, therefore,

$$
\begin{aligned}
P\left\{\left|\left\langle\tilde{S}_{T}, \Omega_{3}\right\rangle\right|\right. & \left.\geqslant 32 K_{0}^{1 / 2} \eta^{-1 / 2}+4\right\} \\
& <P\left\{\left|\tilde{S}_{T}\right| \geqslant 32 K_{0}^{1 / 2} \eta^{-1 / 2}+4\right\}<\eta / 8<\frac{1}{2} .
\end{aligned}
$$

In particular, we must have

$$
|\nu| \leqslant K_{16}=\left(32 K_{0}^{1 / 2} \eta^{-1 / 2}+4\right) \quad(\operatorname{see}(3.39)) .
$$

We can now complete the proof of the lemma by means of an application of Corollary 2. By (3.43) either $\mu>0$ or $\mu<0$. For the sake of argument take $\mu>0$. Then (3.42) and (3.43) give

$$
\begin{aligned}
P\left\{K_{17} / 4<\mu / 2<\mu-8 \varepsilon_{1} \eta^{-1 / 2}\right. & \left.<\left\langle\tilde{S}_{T}, \Omega_{1}\right\rangle<\mu+8 \varepsilon_{1} \eta^{-1 / 2}\right\} \\
& >1-\eta / 16 .
\end{aligned}
$$

(3.59) and (3.55) yield

$$
\begin{aligned}
P\left\{\left\langle\tilde{S}_{T}, \Omega_{1}\right\rangle>\frac{1}{4} K_{17},\left\langle\tilde{S}_{T}, \Omega_{3}\right\rangle<\left(\nu+\frac{1}{8} K_{17}\right) \mu^{-1}\left\langle\tilde{S}_{T}, \Omega_{1}\right\rangle\right\} \\
\quad>P\left\{\mu-8 \varepsilon_{1} \eta^{-1 / 2}<\left\langle\tilde{S}_{T}, \Omega_{1}\right\rangle<\mu+8 \varepsilon_{1} \eta^{-1 / 2},\left\langle\tilde{S}_{T}, \Omega_{3}\right\rangle<\nu\right\} \\
\quad>P\left\{\left\langle\tilde{S}_{T}, \Omega_{3}\right\rangle<\nu\right\}-\frac{\eta}{16}>\frac{1}{2}-\frac{\eta}{16}>\frac{1}{4} .
\end{aligned}
$$

In exactly the same way one obtains from (3.59) and (3.53):

$$
\begin{aligned}
P & \left\{\left\langle\tilde{S}_{T}, \Omega_{1}\right\rangle \geqslant \frac{1}{4} K_{17},\left\langle\tilde{S}_{T}, \Omega_{3}\right\rangle \geqslant\left(\nu+\frac{1}{8} K_{17}\right) \mu^{-1}\left\langle\tilde{S}_{T}, \Omega_{1}\right\rangle+\frac{1}{8} K_{17}\right\} \\
& >P\left\{\mu-8 \varepsilon_{1} \eta^{-1 / 2}<\left\langle\tilde{S}_{T}, \Omega_{1}\right\rangle<\mu+8 \varepsilon_{1} \eta^{-1 / 2},\left\langle\tilde{S}_{T}, \Omega_{3}\right\rangle>\nu+\frac{1}{2} K_{17}\right\} \\
& >\eta / 8-\eta / 16=\eta / 16 .
\end{aligned}
$$

Lastly, we observe that

$$
\begin{aligned}
P\left\{\tilde{Y}_{1} \neq \tilde{S}_{T}\right\} & =P\left\{\sum_{1}^{\nu_{1}} X_{n} \neq \sum_{1}^{T} X_{n}\right\}=P\left\{\nu_{1} \neq T\right\} \\
& <P\left\{J_{n}=1 \text { for some } n<T\right\}<\frac{1}{32} \eta \quad \text { (by (3.33)), }
\end{aligned}
$$


so that with

$$
d_{1}=\frac{1}{4} K_{17}, \quad d_{2}=\left(\nu+\frac{1}{8} K_{17}\right) \mu^{-1}, \quad d_{3}=\frac{1}{8} K_{17}
$$

(3.60) and (3.61) give

$$
P\left\{\left\langle\tilde{Y}_{1}, \Omega_{1}\right\rangle>d_{1},\left\langle\tilde{Y}_{1}, \Omega_{3}\right\rangle\left\langle d_{2}\left\langle\tilde{Y}_{1}, \Omega_{1}\right\rangle\right\}>1 / 4-\eta / 32>1 / 8,\right.
$$

and

$$
P\left\{\left\langle\tilde{Y}_{1}, \Omega_{1}\right\rangle>d_{1},\left\langle\tilde{Y}_{1}, \Omega_{3}\right\rangle \geqslant d_{2}\left\langle\tilde{Y}_{1}, \Omega_{1}\right\rangle+d_{3}\right\} \geqslant \eta / 16-\eta / 32=2^{-5} \eta .
$$

These inequalities are just (3.8) and (3.9) for $Z_{1}(1)=\left\langle\tilde{Y}_{1}, \Omega_{3}\right\rangle, Z_{1}(2)=$ $\left\langle\tilde{Y}_{1}, \Omega_{1}\right\rangle$ and $p_{3}=\frac{1}{8}, p_{4}=2^{-5} \eta$. Moreover, $d_{3} d_{1}^{-1}, p_{3}, p_{4}$ all have strictly positive lower bounds which are independent of $F$, whereas (see (3.43) and (3.58))

$$
\left|d_{2}\right|<\left(K_{16}+\frac{1}{8} K_{17}\right)|\mu|^{-1}<2\left(K_{16}+\frac{1}{8} K_{17}\right) K_{17}^{-1} \text {. }
$$

It follows there from (3.10) and (3.11) that for some $K_{18}<\infty$ and all $s>1$, (3.36) is bounded by

$$
\begin{gathered}
E \#\left\{r \in[s, 2 s]:\left|\sum_{n=1}^{r}\left\{\left\langle\tilde{Y}_{n}, \Omega_{1}\right\rangle \Omega_{1}+\left\langle\tilde{Y}_{n}, \Omega_{3}\right\rangle \Omega_{3}\right\}\right|<8\right\} \\
\quad \text { (because } \Omega_{1}, \Omega_{3} \text { is an orthonormal basis of } \mathcal{H C} \text { ) } \\
<K_{18}\left\{\left(32 K_{17}^{-1}\right)^{2}+1\right\} .
\end{gathered}
$$

This gives the desired bound for (3.36) and (3.1) under (3.33).

LEMMA 5. There exist a $k_{7}=k_{7}(F)<\infty$ and $K_{19}<\infty$ such that the left-hand side of (3.1) is bounded by $K_{19}$ for all $k>k_{7}(F)$ for which (3.34) holds.

Proof. Set

$$
\tilde{W}_{r}=\sum_{n=\nu_{r-1}+1}^{\nu_{r}}\left\{\left\langle\tilde{X}_{n} J_{n}, \omega_{1}\right\rangle \omega_{1}+\left\langle\tilde{X}_{n} J_{n}, \omega_{2}\right\rangle \omega_{2}\right\} .
$$

As mentioned in (3.37) and the lines following it, $\tilde{Y}_{r}=\tilde{Z}_{r}+\tilde{W}_{r}$ and all $\tilde{Z}_{r}, \tilde{W}_{s}, r>1, s \geqslant 1$, are independent. Just as in (3.38) the two dimensional analogue of (2.32) therefore gives for any unit vectors $\Omega_{i} \in \mathcal{H}$ and $x_{i}>0$, $i \in I$ (some finite set of positive integers)

$$
\begin{aligned}
\sup _{z} P\left\{\left|\left\langle\sum_{n=1}^{r} \tilde{Y}_{n}, \Omega_{i}\right\rangle+z_{i}\right| \leqslant x_{i}, i \in I\right\} \\
\quad<\sup _{z} P\left\{\left|\left\langle\sum_{n=1}^{r} \tilde{W}_{n}, \Omega_{i}\right\rangle+z_{i}\right| \leqslant x_{i}, i \in I\right\} .
\end{aligned}
$$


The distribution of

$$
W_{r}=\sum_{n=\nu_{r-1}+1}^{\nu_{r}} X_{n} J_{n}
$$

was given in (2.49) and (2.50). As there we take $\alpha_{1}, \alpha_{2}, \ldots$ independent and each with the conditional distribution of $X_{1}$ given $J_{1}=1$. Then $\tilde{\alpha}_{i}$ has the conditional distribution of $\tilde{X}_{1}$ given $J_{1}=1$ and $\tilde{W}_{r}$ the distribution of $\sum_{i=1}^{\Lambda} \tilde{\alpha}_{i}$. Moreover, by (2.46),

$$
\begin{aligned}
& P\left\{\left|\left\langle\tilde{\alpha}_{i}, \omega_{1}\right\rangle\right|>1 \text { or }\left|\left\langle\tilde{\alpha}_{1}, \omega_{2}\right\rangle\right|>1\right\} \\
& \quad=P\left\{\left|\left\langle X_{1}, \omega_{1}\right\rangle\right|>m(k) A_{k} \text { or }\left|\left\langle X_{1}, \omega_{2}\right\rangle\right|>A_{k} \mid J_{1}=1\right\}=1,
\end{aligned}
$$

and a fortiori

$$
P\left\{\left|\tilde{\alpha}_{i}\right|>1\right\}=1 .
$$

Now consider the unit vectors

$$
u_{l}=\left(\cos (2 l+1) \frac{\pi}{16}\right) \omega_{1}+\left(\sin (2 l+1) \frac{\pi}{16}\right) \omega_{2}, \quad l=0, \ldots, 7,
$$

all of which lie in $\mathcal{X} \cap\left\{z \in \mathbf{R}^{2}: z(2)>0\right\}$, and are such that every vector in $\mathcal{H}$ makes an angle at most $\pi / 16$ with some $u_{l}$ or $-u_{l}$. Thus one can choose a $0<l<7$ such that

$$
P\left\{\varphi\left(\tilde{\alpha}_{1}, u_{l}\right)<\frac{\pi}{16} \text { or } \varphi\left(\tilde{\alpha}_{1},-u_{1}\right)<\frac{\pi}{16}\right\}>\frac{1}{8} .
$$

With $l$ fixed in this way one can also find two orthogonal unit vectors $\Omega_{1}, \Omega_{2}$ in $\mathcal{H}$ such that $\varphi\left(\Omega_{1}, u_{l}\right)<\pi / 16$,

$$
\begin{aligned}
P\left\{\varphi\left(\tilde{\alpha}_{1}, \Omega_{1}\right)<\pi / 8 \text { or } \varphi\left(\tilde{\alpha}_{1},-\Omega_{1}\right)\right. & <\pi / 8, \\
& \left.\left\langle\tilde{\alpha}_{1}, \Omega_{1}\right\rangle \cdot\left\langle\tilde{\alpha}_{1}, \Omega_{2}\right\rangle \geqslant 0\right\}>1 / 16
\end{aligned}
$$

as well as

$$
\begin{aligned}
& P\left\{\varphi\left(\tilde{\alpha}_{1}, \Omega_{1}\right) \leqslant \pi / 8 \text { or } \varphi\left(\tilde{\alpha}_{1},-\Omega_{1}\right)\right.<\pi / 8, \\
&\left.\left\langle\tilde{\alpha}_{1}, \Omega_{1}\right\rangle\left\langle\tilde{\alpha}_{1}, \Omega_{2}\right\rangle<0\right\}>1 / 16 .
\end{aligned}
$$

Note that (3.66) merely says that there is a probability at least $\frac{1}{16}$ for $\tilde{\alpha}_{1}$ to lie in the first or third quadrant with respect to the $\Omega_{1}, \Omega_{2}$ axes and even within $\pi / 8$ from the positive or negative $\Omega_{1}$ axis. (3.67) says the same thing with first and third quadrant replaced by second and fourth quadrant; such $\Omega_{1}, \Omega_{2}$ are easily obtained by continuity considerations, for if one chooses $\Omega_{1}$ first along one boundary line of the set in braces in (3.65) then there is probability at least $\frac{1}{8}$ that $\tilde{\alpha}_{1}$ lies in the first or third quadrant, and when $\Omega_{1}$ is rotated to the other boundary line then one ends up with probability at least $\frac{1}{8}$ that $\tilde{\alpha}_{1}$ lies in the second or fourth quadrant. Note that $\Omega_{1}, \Omega_{2}$ really depend on $k$, since $\tilde{\alpha}_{1}$ does. The same holds for $\varphi_{1}$ and $\omega_{0}$ below, but this will not influence the 
sequel. Assume

$$
\Omega_{1}=\left(-\sin \varphi_{1}\right) \omega_{1}+\left(\cos \varphi_{1}\right) \omega_{2}, \quad \Omega_{2} \text { or }-\Omega_{2}=\left(\cos \varphi_{1}\right) \omega_{1}+\left(\sin \varphi_{1}\right) \omega_{2}
$$

and fix $\omega_{0}$, a unit vector in $\mathcal{K}, K_{20}<\infty$ and $\varepsilon_{2}>0$ as follows:

$$
\begin{aligned}
\omega_{0}= & \left\{m(k)^{-2} \cos ^{2} \varphi_{1}+\sin ^{2} \varphi_{1}\right\}^{-1 / 2} \\
& \cdot\left\{m(k)^{-1}\left(\cos \varphi_{1}\right) \omega_{1}+\left(\sin \varphi_{1}\right) \omega_{2}\right\}, \\
L_{0}= & {\left[32 K_{0} \eta^{-1}\right]+1, \quad x_{0}=24 K_{0} \eta^{-1}+1, } \\
K_{20}= & \min \left\{\left(64 L_{0}\right)^{-1}, 2^{-7}\left(12 x_{0}+3\right)^{-1}\right\}, \quad \varepsilon_{2}=\left(32 L_{0}\right)^{-1} \eta .
\end{aligned}
$$

For brevity we shall write

$$
\Theta=\Theta(k)=\left\{m(k)^{-2} \cos ^{2} \varphi_{1}+\sin ^{2} \varphi_{1}\right\}^{1 / 2} .
$$

We consider three separate cases now. Which case we are in depends on which of the inequalities (3.71)-(3.73) holds:

$$
\begin{array}{r}
P\left\{\left|\left\langle\tilde{\alpha}_{1}, \Omega_{2}\right\rangle\right|>\frac{1}{2}\right\}>\varepsilon_{2}, \\
P\left\{K_{20} M\left(\omega_{0}, k\right) \Theta(k)<\left|\left\langle\tilde{\alpha}_{1}, \Omega_{2}\right\rangle\right| \leqslant \frac{1}{2}\right\}>\varepsilon_{2}, \\
P\left\{\left|\left\langle\tilde{\alpha}_{1}, \Omega_{2}\right\rangle\right|>K_{20} M\left(\omega_{0}, k\right) \Theta(k)\right\}<2 \varepsilon_{2} .
\end{array}
$$

First assume (3.71); without loss of generality we may then assume (if necessary replace $\Omega_{2}$ by $-\Omega_{2}$ and /or $\Omega_{1}$ by $-\Omega_{1}$ ) that

$$
P\left\{\left\langle\tilde{\alpha}_{1}, \Omega_{2}\right\rangle>\frac{1}{2},\left\langle\tilde{\alpha}_{1}, \Omega_{1}\right\rangle>0\right\}>\frac{1}{4} \varepsilon_{2} .
$$

In this case we take

$$
\Omega_{3}=\frac{1}{\sqrt{2}}\left\{-\Omega_{1}+\Omega_{2}\right\}, \quad \Omega_{4}=\frac{1}{\sqrt{2}}\left\{\Omega_{1}+\Omega_{2}\right\} .
$$

$\Omega_{3}$ and $\Omega_{4}$ are orthogonal unit vectors, bisecting the second (resp. first) quadrant with respect to $\Omega_{1}, \Omega_{2}$. Obviously $\left\langle\tilde{\alpha}_{1}, \Omega_{2}\right\rangle>\frac{1}{2},\left\langle\tilde{\alpha}_{1}, \Omega_{1}\right\rangle>0$ entails

$$
\left.\left\langle\tilde{\alpha}_{1}, \Omega_{3}\right\rangle=-\left\langle\tilde{\alpha}_{1}, \Omega_{4}\right\rangle+\sqrt{2}\left\langle\tilde{\alpha}_{1}, \Omega_{2}\right\rangle\right\rangle-\left\langle\tilde{\alpha}_{1}, \Omega_{4}\right\rangle+1 / \sqrt{2}
$$

as well as

$$
\left\langle\tilde{\alpha}_{1}, \Omega_{4}\right\rangle>2^{-3 / 2}
$$

Thus (3.74) implies

$$
P\left\{\left\langle\tilde{\alpha}_{1}, \Omega_{4}\right\rangle>2^{-3 / 2},\left\langle\tilde{\alpha}_{1}, \Omega_{3}\right\rangle \geqslant-\left\langle\tilde{\alpha}_{1}, \Omega_{4}\right\rangle+2^{-1 / 2}\right\} \geqslant \frac{1}{4} \varepsilon_{2} .
$$

But also, from (3.64) and (3.67), one has

$$
P\left\{\left\langle\tilde{\alpha}_{1}, \Omega_{4}\right\rangle \geqslant \cos \frac{3 \pi}{8},\left\langle\tilde{\alpha}_{1}, \Omega_{3}\right\rangle\left\langle-\left\langle\tilde{\alpha}_{1}, \Omega_{4}\right\rangle\right\} \geqslant \frac{1}{32}\right.
$$

or the same inequality with $\tilde{\alpha}_{1}$ replaced by $-\tilde{\alpha}_{1}$. Thus if we put 


$$
d_{1}=\frac{1}{3}, \quad d_{2}=-1, \quad d_{3}=2^{-1 / 2}, \quad p_{3}=\frac{1}{32}, \quad p_{4}=\frac{1}{4} \varepsilon_{2},
$$

then (3.9) holds with $Z_{1}(1)=\left\langle\tilde{\alpha}_{1}, \Omega_{3}\right\rangle, Z_{1}(2)=\left\langle\tilde{\alpha}_{1}, \Omega_{4}\right\rangle$, and (3.8) holds with the same replacement or with $-Z_{1}(1)=\left\langle\tilde{\alpha}_{1}, \Omega_{3}\right\rangle,-Z_{1}(2)=\left\langle\tilde{\alpha}_{1}, \Omega_{4}\right\rangle$. Moreover, by (3.34) and (2.29) we also have (3.12) with $d_{4}=2^{-5} \eta, d_{5}=2 K_{0}$ as soon as $k>k_{7}$ for suitable $k_{7}=k_{7}(F)<\infty$. Thus, by (3.63) and (3.13),

$$
\begin{aligned}
\sup _{z} P\left\{\left|\sum_{n=1}^{r}\left\langle\tilde{Y}_{n}, \omega_{j}\right\rangle+z_{j}\right| \leqslant 4, j=1,2\right\} \\
\quad \sup _{z} P\left\{\left|\sum_{n=1}^{r} \tilde{W}_{n}+z\right| \leqslant 8\right\}<K_{21} r^{-1}
\end{aligned}
$$

for some $K_{21}<\infty$ depending on $\varepsilon_{2}, \eta$ and $K_{0}$ only. Thus $K_{21}$ does not depend on $F$, and in the case where (3.71) holds we obtain the bound

$$
\sum_{r=s}^{2 s} K_{21} r^{-1} \leqslant K_{21}(1+\log 2)
$$

for (3.36) and the left-hand side of (3.1).

Next we consider the case where (3.72) holds. Again we assume that the signs of $\Omega_{1}, \Omega_{2}$ have been chosen such that

$$
P\left\{K_{20} M\left(\omega_{0}, k\right) \Theta(k)<\left\langle\tilde{\alpha}_{1}, \Omega_{2}\right\rangle\left\langle\frac{1}{2},\left\langle\tilde{\alpha}_{1}, \Omega_{1}\right\rangle>0\right\}>\frac{1}{4} \varepsilon_{2}\right.
$$

(compare (3.74)). Since $\left|\tilde{\alpha}_{1}\right|>1$ w.p.1 (see (3.64)), $\left|\left\langle\tilde{\alpha}_{1}, \Omega_{2}\right\rangle\right|<\frac{1}{2}$ implies $\left|\left\langle\tilde{\alpha}_{1}, \Omega_{1}\right\rangle\right|>\frac{1}{2}$. Thus, if we define for any random vector $X$,

$$
\begin{aligned}
\bar{X} & =\left\langle\tilde{X}, \Omega_{1}\right\rangle \Omega_{1}+\left\{M\left(\omega_{0}, k\right) \Theta(k)\right\}^{-1}\left\langle\tilde{X}, \Omega_{2}\right\rangle \Omega_{2} \\
& =\left\langle\tilde{X}, \Omega_{1}\right\rangle \Omega_{1}+M\left(\omega_{0}, k\right)^{-1}\left\{m(k)^{-2} \cos ^{2} \varphi_{1}+\sin ^{2} \varphi_{1}\right\}^{-1 / 2}\left\langle\tilde{X}, \Omega_{2}\right\rangle \Omega_{2},
\end{aligned}
$$

then (3.76) implies

$$
P\left\{\left\langle\bar{\alpha}_{1}, \Omega_{1}\right\rangle=\left\langle\tilde{\alpha}_{1}, \Omega_{1}\right\rangle>\frac{1}{2},\left\langle\bar{\alpha}_{1}, \Omega_{2}\right\rangle>K_{20}\right\}>\frac{1}{4} \varepsilon_{2} .
$$

Also, (3.67) together with (3.64) gives

$$
\begin{aligned}
& P\left\{\left|\left\langle\bar{\alpha}_{1}, \Omega_{1}\right\rangle\right| \geqslant \cos \frac{\pi}{8},\left\langle\bar{\alpha}_{1}, \Omega_{1}\right\rangle\left\langle\bar{\alpha}_{1}, \Omega_{2}\right\rangle \leqslant 0\right\} \\
& \geqslant P\left\{\left|\tilde{\alpha}_{1}\right|>1, \varphi\left(\tilde{\alpha}_{1}, \Omega_{1}\right) \leqslant \pi / 8\right. \\
& \left.\quad \text { or } \varphi\left(\tilde{\alpha}_{1},-\Omega_{1}\right) \leqslant \pi / 8,\left\langle\tilde{\alpha}_{1}, \Omega_{1}\right\rangle\left\langle\tilde{\alpha}, \Omega_{2}\right\rangle \leqslant 0\right\}>1 / 16,
\end{aligned}
$$

so that

$$
P\left\{\left\langle\bar{\alpha}_{1}, \Omega_{1}\right\rangle \geqslant \cos \frac{\pi}{8},\left\langle\bar{\alpha}_{1}, \Omega_{2}\right\rangle \leqslant 0\right\} \geqslant \frac{1}{32}
$$

or 


$$
P\left\{\left\langle-\bar{\alpha}_{1}, \Omega_{1}\right\rangle>\cos \frac{\pi}{8},\left\langle-\bar{\alpha}_{1}, \Omega_{2}\right\rangle\langle 0\}>\frac{1}{32} .\right.
$$

If we take

$$
d_{1}=\frac{1}{2}, \quad d_{2}=0, \quad d_{3}=K_{20}, \quad p_{3}=\frac{1}{32}, \quad p_{4}=\frac{1}{4} \varepsilon_{2},
$$

then (3.77) is again (3.9) with $Z_{1}(1)=\left\langle\bar{\alpha}_{1}, \Omega_{2}\right\rangle, Z_{1}(2)=\left\langle\bar{\alpha}_{1}, \Omega_{1}\right\rangle$; (3.78) becomes (3.8) and (3.79) is (3.8) with $Z$ replaced by $-Z$. As before we also have (3.12) with $d_{4}=2^{-5} \eta$ and $d_{5}=2 K_{0}$ for $k>k_{7}$ from (3.34) and (2.29). Thus, by (3.13) and (3.63),

$$
\begin{gathered}
\sup _{z} P\left\{\left|\sum_{n=1}^{r}\left\langle\bar{Y}_{n}, \Omega_{j}\right\rangle+z_{j}\right| \leqslant 8, j=1,2\right\} \\
\quad \sup _{z} P\left\{\left|\sum_{n=1}^{r}\left\langle\bar{W}_{n}, \Omega_{j}\right\rangle+z_{j}\right| \leqslant 8, j=1,2\right\} \\
<\sup _{z} P\left\{\left|\sum_{n=1}^{r} \bar{W}_{n}+z\right| \leqslant 16\right\} \leqslant K_{22} r^{-1} .
\end{gathered}
$$

Again $K_{22}<\infty$ depends neither on $F$ nor $k$. However,

$$
\begin{aligned}
\sum_{n=1}^{r}\left\langle\bar{Y}_{n}, \Omega_{1}\right\rangle & =\sum_{n=1}^{r}\left\langle\tilde{Y}_{n}, \Omega_{1}\right\rangle \\
= & A_{k}^{-1}\left\{-\sum_{n=1}^{r}\left\langle Y_{n}, \omega_{1}\right\rangle \frac{1}{m(k)} \sin \varphi_{1}+\sum_{n=1}^{r}\left\langle Y_{n}, \omega_{2}\right\rangle \cos \varphi_{1}\right\}
\end{aligned}
$$

and

$$
\begin{aligned}
\left|\sum_{n=1}^{r}\left\langle\bar{Y}_{n}, \Omega_{2}\right\rangle\right|= & \left\{M\left(\omega_{0}, k\right) \Theta(k)\right\}^{-1}\left|\sum_{n=1}^{r}\left\langle\tilde{Y}_{n}, \Omega_{2}\right\rangle\right| \\
= & \left\{A_{k} M\left(\omega_{0}, k\right) \Theta(k)\right\}^{-1} \\
& \cdot\left|\sum_{n=1}^{r}\left\langle Y_{n}, \omega_{1}\right\rangle \frac{1}{m(k)} \cos \varphi_{1}+\sum_{n=1}^{r}\left\langle Y_{n}, \omega_{2}\right\rangle \sin \varphi_{1}\right| \\
= & A_{k}^{-1} M\left(\omega_{0}, k\right)^{-1}\left|\sum_{n=1}^{r}\left\langle Y_{n}, \omega_{0}\right\rangle\right| .
\end{aligned}
$$

It follows that the condition

$$
\left|\sum_{n=1}^{r}\left\langle Y_{n}, \omega_{j}\right\rangle\right| \leqslant 4 M\left(\omega_{j}, k\right) A_{k}
$$

for $j=0$ is equivalent to 


$$
\left|\sum_{n=1}^{r}\left\langle\bar{Y}_{n}, \Omega_{2}\right\rangle\right|<4,
$$

and condition (3.83) for $j=1$ and 2 implies

$$
\left|\sum_{n=1}^{r}\left\langle\bar{Y}_{n}, \Omega_{1}\right\rangle\right|<4\left|\sin \varphi_{1}\right|+4\left|\cos \varphi_{1}\right|<8
$$

(recall $\left.M\left(\omega_{1}, k\right)=m(k), M\left(\omega_{2}, k\right)=1\right)$. Thus, (3.1) is bounded by

$$
\begin{aligned}
\sum_{r=s}^{2 s} \sup _{z} P\left\{\left|\sum_{n=1}^{r}\left\langle\bar{Y}_{n}, \Omega_{j}\right\rangle+z_{j}\right|<8, j=1,2\right\} \\
\quad<\sum_{r=s}^{2 s} K_{22} r^{-1}(\operatorname{see}(3.80))<K_{22}(1+\log 2) .
\end{aligned}
$$

This settles the case where (3.72) holds and we are left with the case where (3.73) holds. Without loss of generality we may also assume that (3.71) fails. We begin with an estimate on the distribution of $\left\langle\tilde{S}_{T}, \Omega_{2}\right\rangle$ in this case. Analogously to (3.82),

$$
\left|\left\langle\tilde{S}_{T}, \Omega_{2}\right\rangle\right|=\Theta(k) A_{k}^{-1}\left|\left\langle S_{T}, \omega_{0}\right\rangle\right|
$$

so that by $(2.30)$,

$$
P\left\{\left|\left\langle\tilde{S}_{T}, \Omega_{2}\right\rangle\right|>\frac{1}{2} \Theta(k) M\left(\omega_{0}, k\right)\right\}>\eta .
$$

Obviously,

$$
\tilde{S}_{T}=\sum_{n=1}^{T} \tilde{X}_{n} J_{n}+\sum_{n=1}^{T} \tilde{X}_{n}\left(1-J_{n}\right) .
$$

Moreover, for any $L>1$,

$$
\begin{aligned}
P\left\{\left|\sum_{n=1}^{T}\left\langle\tilde{X}_{n} J_{n}, \Omega_{2}\right\rangle\right|>\frac{1}{4} \Theta(k) M\left(\omega_{0}, k\right)\right\} \\
\quad<P\left\{\sum_{n=1}^{T} J_{n}>L\right\}+P\left\{\sum_{i=1}^{L}\left|\left\langle\tilde{\alpha}_{i}, \Omega_{2}\right\rangle\right|>\frac{1}{4} \Theta(k) M\left(\omega_{0}, k\right)\right\} ;
\end{aligned}
$$

indeed once we know that $J_{n}=1$ exactly for $n \in\left\{n_{1}, \ldots, n_{L}\right\}$ and no other $n \in[1, T]$, the conditional distribution of

$$
\sum_{n=1}^{T} \tilde{X}_{n} J_{n}
$$

is simply the distribution of

$$
\sum_{i=1}^{L} \tilde{\alpha}_{i}
$$


(compare (2.49) and (2.50)). The right-hand side of (3.85) is bounded by

$$
\frac{T}{L} E J_{1}+L P\left\{\left|\left\langle\tilde{\alpha}_{1}, \Omega_{2}\right\rangle\right| \geqslant \frac{1}{4 L} \Theta(k) M\left(\omega_{0}, k\right)\right\} .
$$

Now take $L=L_{0}$ (see (3.69)). Then it follows from (2.53), (3.70) and (3.73) that (3.85) is at most

$$
\begin{aligned}
\left(T / L_{0}\right) P\left\{J_{1}=1\right\}+L_{0} P\left\{\left|\left\langle\tilde{\alpha}_{1}, \Omega_{2}\right\rangle\right|>K_{20} \Theta M\left(\omega_{0}, k\right)\right\} \\
\\
<\left(2 / L_{0}\right) K_{0}+L_{0} 2 \varepsilon_{2}<\eta / 8 .
\end{aligned}
$$

When this bound for (3.85) is combined with (3.84) we find

$$
P\left\{\left|\sum_{n=1}^{T}\left\langle\tilde{X}_{n}\left(1-J_{n}\right), \Omega_{2}\right\rangle\right| \geqslant \frac{1}{4} \Theta(k) M\left(\omega_{0}, k\right)\right\} \geqslant \frac{7}{8} \eta .
$$

Next we need an estimate for the distribution of

$$
\sum_{n=1}^{T}\left\langle\tilde{X}_{n}\left(1-J_{n}\right), \omega_{j}\right\rangle, \quad j=1,2,
$$

which is a slight variation on (3.56). E.g., take $j=1$ and write temporarily

$$
I_{n}=I\left[\left|\left\langle X_{n}, \omega_{1}\right\rangle\right| \leqslant m(k) A_{k}\right]=I\left[\left|\left\langle\tilde{X}_{n}, \omega_{1}\right\rangle\right|<1\right] .
$$

Then, as in (3.56), for $k \geqslant k_{3}(F)$,

$$
\begin{aligned}
P\left\{\left|\sum_{n=1}^{T}\left\langle\tilde{X}_{n} I_{n}, \omega_{1}\right\rangle-T E\left\langle\tilde{X}_{1}, \omega_{1}\right\rangle I_{1}\right| \geqslant x\right\} \\
\quad<\left(T(k) / x^{2}\right) \sigma^{2}\left\{\left\langle\tilde{X}_{1}, \omega_{1}\right\rangle I\left[\left|\left\langle X_{1}, \omega_{1}\right\rangle\right| \leqslant m(k) A_{k}\right]\right\} \leqslant K_{0} / x^{2}
\end{aligned}
$$

But also

$$
I_{n}-\left(1-J_{n}\right)=I\left[\left|\left\langle\tilde{X}_{n}, \omega_{1}\right\rangle\right|<1,\left|\left\langle\tilde{X}_{n}, \omega_{2}\right\rangle\right|>1\right]
$$

so that

$$
\begin{aligned}
\left|\sum_{n=1}^{T}\left\langle\tilde{X}_{n}\left(1-J_{n}\right), \omega_{1}\right\rangle-\sum_{n=1}^{T}\left\langle\tilde{X}_{n} I_{n}, \omega_{1}\right\rangle\right| \\
\quad<\sum_{n=1}^{T}\left|\left\langle\tilde{X}_{n}, \omega_{1}\right\rangle\right|\left|1-J_{n}-I_{n}\right| \\
<\#\left\{n<T:\left|\left\langle\tilde{X}_{n}, \omega_{1}\right\rangle\right|<1,\left|\left\langle\tilde{X}_{n}, \omega_{2}\right\rangle\right|>1\right\} \\
\quad<\sum_{n=1}^{T} J_{n} .
\end{aligned}
$$

It follows that 


$$
\begin{gathered}
P\left\{\left|\sum_{n=1}^{T}\left\langle\tilde{X}_{n}\left(1-J_{n}\right), \omega_{1}\right\rangle-\sum_{n=1}^{T}\left\langle\tilde{X}_{n} I_{n}, \omega_{1}\right\rangle\right|>x\right\} \\
<x^{-1} T(k) E J_{1}<2 x^{-1} K_{0} \quad(\text { by (2.53)). }
\end{gathered}
$$

Combined with (3.89) this estimate yields

$$
\begin{gathered}
P\left\{\left|\sum_{n=1}^{T}\left\langle\tilde{X}_{n}\left(1-J_{n}\right), \omega_{1}\right\rangle-T E\left\langle\tilde{X}_{1}, \omega_{1}\right\rangle I\left[\left|\left\langle\tilde{X}_{1}, \omega_{1}\right\rangle\right|<1\right]\right|>2 x\right\} \\
<K_{0}\left(2 x^{-1}+x^{-2}\right) .
\end{gathered}
$$

This inequality remains valid when $\omega_{1}$ is replaced by $\omega_{2}$ throughout and thus, if we define

$$
\begin{gathered}
\rho=\rho(k)=-E\left\langle\tilde{X}_{1}, \omega_{1}\right\rangle I\left[\left|\left\langle\tilde{X}_{1}, \omega_{1}\right\rangle\right|<1\right] \sin \varphi_{1} \\
+E\left\langle\tilde{X}_{1}, \omega_{2}\right\rangle I\left[\left|\left\langle\tilde{X}_{1}, \omega_{2}\right\rangle\right|<1\right] \cos \varphi_{1},
\end{gathered}
$$

then

$$
P\left\{\left|\sum_{n=1}^{T}\left\langle\tilde{X}_{n}\left(1-J_{n}\right), \Omega_{1}\right\rangle-T(k) \rho(k)\right|>4 x\right\}<2 K_{0}\left(2 x^{-1}+x^{-2}\right) .
$$

Now observe that by (2.21) and (2.24),

$$
\begin{aligned}
P\left\{\left|\left\langle\tilde{S}_{T}, \Omega_{1}\right\rangle\right|<3\right\} & >P\left\{\left|\left\langle\tilde{S}_{T}, \omega_{1}\right\rangle\right|<1,\left|\left\langle\tilde{S}_{T}, \omega_{2}\right\rangle\right|<2\right\} \\
& >1-P\left\{\left|\left\langle S_{T}, \omega_{1}\right\rangle\right|>m(k) A_{k}\right\}-P\left\{\left|\left\langle S_{T}, \omega_{2}\right\rangle\right|>2 A_{k}\right\} \\
& >1-3(8 d+8)^{-1}>\frac{1}{2} .
\end{aligned}
$$

Thus, for $x_{0}$ as in (3.69) we have

$$
2 K_{0}\left(2 x_{0}^{-1}+x_{0}^{-2}\right)<\eta / 4<1 / 4 \text {, }
$$

and

$$
\begin{aligned}
& P\left\{\left|\sum_{n=1}^{T}\left\langle\tilde{X}_{n} J_{n}, \Omega_{1}\right\rangle+T(k) \rho(k)\right| \leqslant 4 x_{0}+3,\right. \\
& \left.\qquad\left|\sum_{n=1}^{T}\left\langle\tilde{X}_{n}\left(1-J_{n}\right), \Omega_{1}\right\rangle-T(k) \rho(k)\right|<4 x_{0}\right\} \\
& >P\left\{\left|\left\langle\tilde{S}_{T}, \Omega_{1}\right\rangle\right|<3,\left|\sum_{n=1}^{T}\left\langle\tilde{X}_{n}\left(1-J_{n}\right), \Omega_{1}\right\rangle-T(k) \rho(k)\right|<4 x_{0}\right\}>1 / 4 .
\end{aligned}
$$

Using the relation between the distributions of (3.86) and (3.87) we obtain 


$$
\begin{aligned}
\frac{1}{4} & <P\left\{\left|\sum_{n=1}^{T}\left\langle\tilde{X}_{n} J_{n}, \Omega_{1}\right\rangle+T(k) \rho(k)\right|<4 x_{0}+3\right\} \\
& <P\left\{\sum_{1}^{T} J_{n}>L_{0}\right\} \\
& +\sum_{l=0}^{L_{0}} P\left\{\sum_{1}^{T} J_{n}=l\right\} P\left\{\left|\sum_{1}^{l}\left\langle\tilde{\alpha}_{i}, \Omega_{1}\right\rangle+T(k) \rho(k)\right|<4 x_{0}+3\right\} \\
< & \frac{\eta}{8}+\max _{l<L_{0}} P\left\{\left|\sum_{1}^{l}\left\langle\tilde{\alpha}_{i}, \Omega_{1}\right\rangle+T(k) \rho(k)\right|<4 x_{0}+3\right\}
\end{aligned}
$$

(compare the estimates for (3.85)). Thus, for some $0<l_{0}<L_{0}$,

$$
P\left\{\left|\sum_{1}^{l_{0}}\left\langle\tilde{\alpha}_{i}, \Omega_{1}\right\rangle+T(k) \rho(k)\right|<4 x_{0}+3\right\}>\frac{1}{8} \text {. }
$$

Similarly we get from (3.88) and (3.90),

$$
\begin{aligned}
& \frac{5}{8} \eta<P\left\{\left|\sum_{n=1}^{T}\left\langle\tilde{X}_{n}\left(1-J_{n}\right), \Omega_{2}\right\rangle\right|>\frac{1}{4} \Theta(k) M\left(\omega_{0}, k\right),\right. \\
&\left.\left|\sum_{n=1}^{T}\left\langle\tilde{X}_{n}\left(1-J_{n}\right), \Omega_{1}\right\rangle-T(k) \rho(k)\right|<4 x_{0}\right\} \\
&< P\left\{\sum_{1}^{T} J_{n}>L_{0}\right\} \\
&+\max _{l<L_{0}} P\left\{\left|\sum_{n=1}^{T-l}\left\langle\tilde{\beta}_{n}, \Omega_{2}\right\rangle\right|>\frac{1}{4} \Theta(k) M\left(\omega_{0}, k\right),\right. \\
&\left.\left|\sum_{n=1}^{T-l}\left\langle\tilde{\beta}_{n}, \Omega_{1}\right\rangle-T(k) \rho(k)\right|<4 x_{0}\right\},
\end{aligned}
$$

where we used the same notation as in (2.47) and (2.48). Again there exists a $0<l_{1}<L_{0}$ such that

$$
\begin{aligned}
P\left\{\left|\sum_{n=1}^{T-l_{1}}\left\langle\tilde{\beta}_{n}, \Omega_{2}\right\rangle\right|>\right. & \frac{1}{4} \Theta(k) M\left(\omega_{0}, k\right), \\
& \left.\left|\sum_{n=1}^{T-l_{1}}\left\langle\tilde{\beta}_{n}, \Omega_{1}\right\rangle-T(k) \rho(k)\right|<4 x_{0}\right\}>\frac{\eta}{2} .
\end{aligned}
$$


But, by (2.29) and (2.53) we can choose $k_{7}(F)<\infty$ such that for $k>k_{7}$,

$$
\begin{aligned}
& P\left\{\left|\sum_{T-l_{1}+1}^{T}\left\langle\tilde{\beta}_{n}, \Omega_{2}\right\rangle\right| \geqslant \frac{1}{8} \Theta(k) M\left(\omega_{0}, k\right)\right\} \\
& \quad=P\left\{\left|\sum_{j=1}^{l_{1}}\left\langle\beta_{j}, \omega_{0}\right\rangle\right| \geqslant \frac{1}{8} M\left(\omega_{0}, k\right) A_{k}\right\} \\
& \quad<l_{1} P\left\{\left|X_{1}\right| \geqslant\left(8 l_{1}\right)^{-1} M\left(\omega_{0}, k\right) A_{k} \mid J_{1}=0\right\} \\
& \quad<l_{1}\left(P\left\{J_{1}=0\right\}\right)^{-1} P\left\{\left|X_{1}\right|>\left(8 l_{1}\right)^{-1} M\left(\omega_{0}, k\right) A_{k}\right\}<\eta / 8 .
\end{aligned}
$$

A similar estimate gives for $k>k_{7}$,

$$
P\left\{\left|\sum_{T-l_{1}+1}^{T}\left\langle\tilde{\beta}_{n}, \Omega_{1}\right\rangle\right|>4 x_{0}\right\}<\frac{\eta}{8},
$$

and we therefore conclude from (3.92) that

$$
\begin{aligned}
P\left\{\left|\sum_{n=1}^{T}\left\langle\tilde{\beta}_{n}, \Omega_{2}\right\rangle\right| \geqslant\right. & \frac{1}{8} \Theta(k) M\left(\omega_{0}, k\right), \\
& \left.\left|\sum_{n=1}^{T}\left\langle\tilde{\beta}_{n}, \Omega_{1}\right\rangle-T(k) \rho(k)\right|<8 x_{0}\right\} \geqslant \frac{\eta}{4} .
\end{aligned}
$$

However, with $\alpha_{i}, \beta_{j}$ and $\Lambda$ independent, $Y_{i}$ has the distribution of $\sum_{n=1}^{T} \beta_{n}+$ $\sum_{i=1}^{\Lambda} \alpha_{i}$ (see (2.46)-(2.50)); (3.91) and (3.93) together therefore give

$$
\begin{gathered}
P\left\{\left|\left\langle\tilde{Y}_{1}, \Omega_{2}\right\rangle\right| \geqslant \frac{1}{16} \Theta(k) M\left(\omega_{0}, k\right),\left|\left\langle\tilde{Y}_{1}, \Omega_{1}\right\rangle\right|<12 x_{0}+3\right\} \\
\geqslant P\left\{\Lambda=l_{0}\right\} P\left\{\left|\sum_{n=1}^{T}\left\langle\tilde{\beta}_{n}, \Omega_{2}\right\rangle\right|>\frac{1}{8} \Theta(k) M\left(\omega_{0}, k\right),\right. \\
\left.\qquad\left|\left\langle\sum_{n=1}^{T} \tilde{\beta}_{n}, \Omega_{1}\right\rangle-T(k) \rho(k)\right|<8 x_{0}\right\} \\
\cdot P\left\{\left|\sum_{i=1}^{l_{0}}\left\langle\tilde{\alpha}_{i}, \Omega_{2}\right\rangle\right| \leqslant \frac{1}{16} \Theta(k) M\left(\omega_{0}, k\right),\right. \\
>P\left\{\Lambda=l_{0}\right\} \cdot \frac{\eta}{4}\left\{\frac{1}{8}-l_{0} P\left\{\left|\left\langle\tilde{\alpha}_{1}, \Omega_{2}\right\rangle\right|>K_{20} \Theta(k) M\left(\omega_{0}, k\right)\right\}\right\} \\
>\frac{1}{64} \eta P\left\{\Lambda=l_{0}\right\} \quad(\operatorname{see}(3.70) \text { and }(3.73)) .
\end{gathered}
$$


Moreover, by (2.50) and (3.34) for any $l$,

$$
\begin{aligned}
P\{\Lambda=l\} & \geqslant(l !)^{-1}\left(T P\left\{J_{1}=1\right\}\right)^{l}\left(1-2 K_{0} T^{-1}\right)^{T} \\
& \geqslant(l !)^{-1}\left(2^{-5} \eta\right)^{l} \exp -4 K_{0},
\end{aligned}
$$

as soon as $K_{0} T(k)^{-1} \leqslant \frac{1}{4}$, i.e., for $k>k_{7}(F)$ if $k_{7}(F)$ is chosen large enough (see (2.29)). Thus, in terms of $\bar{Y}_{1}$, (3.94) yields for $k>k_{7}$ and some $K_{23}>0$, independent of $F$ and $k$,

$$
\begin{aligned}
P\left\{\nu_{1}=T+l_{0},\left|\left\langle\bar{Y}_{1}, \Omega_{2}\right\rangle\right| \geqslant \frac{1}{16},\right. \\
\left.\left|\left\langle\bar{Y}_{1}, \Omega_{1}\right\rangle\right|<16\left(12 x_{0}+3\right)\left|\left\langle\bar{Y}_{1}, \Omega_{2}\right\rangle\right|\right\} \geqslant K_{23} .
\end{aligned}
$$

We can therefore find a number $x_{1}=x_{1}(F, k),\left|x_{1}\right|<16\left(12 x_{0}+3\right)$ such that

$$
P\left\{\nu_{1}=T+l_{0},\left\langle\bar{Y}_{1}, \Omega_{2}\right\rangle \geqslant \frac{1}{16},\left\langle\bar{Y}_{1}, \Omega_{1}\right\rangle<x_{1}\left\langle\bar{Y}_{1}, \Omega_{2}\right\rangle\right\} \geqslant \frac{1}{4} K_{23},
$$

as well as

$$
\begin{aligned}
P\left\{\nu_{1}=T+\right. & l_{0},\left\langle\bar{Y}_{1}, \Omega_{2}\right\rangle \geqslant \frac{1}{16}, \\
& \left.\left\langle\bar{Y}_{1}, \Omega_{1}\right\rangle \geqslant x_{1}\left\langle\bar{Y}_{1}, \Omega_{2}\right\rangle\right\}>\frac{1}{4} K_{23},
\end{aligned}
$$

or both of these inequalities hold with $\left\langle\bar{Y}_{1}, \Omega_{2}\right\rangle$ replaced by $-\left\langle\bar{Y}_{1}, \Omega_{2}\right\rangle$. By changing the sign of $\Omega_{2}$, if necessary, we may restrict ourselves to the case where (3.95) and (3.96) hold. Lastly, we observe that (3.64), (3.70), the inequality $\left|x_{1}\right| K_{20} \leqslant 16\left(12 x_{0}+3\right) K_{20}<\frac{1}{4},(3.73)$ and the fact that (3.71) fails imply

$$
\begin{aligned}
P\{ & \left.\left|\left\langle\bar{\alpha}_{l_{0}+1}, \Omega_{2}\right\rangle\right|<K_{20},\left|\left\langle\bar{\alpha}_{l_{0}+1}, \Omega_{1}\right\rangle\right| \geqslant\left|x_{1}\left\langle\bar{\alpha}_{l_{0}+1}, \Omega_{2}\right\rangle\right|+\frac{1}{4}\right\} \\
& \geqslant P\left\{\left|\left\langle\tilde{\alpha}_{l_{0}+1}, \Omega_{2}\right\rangle\right|<\min \left\{\frac{1}{2}, K_{20} \Theta(k) M\left(\omega_{0}, k\right)\right\},\left|\left\langle\bar{\alpha}_{l_{0}+1}, \Omega_{1}\right\rangle\right| \geqslant \frac{1}{2}\right\} \\
& >P\left\{\left|\left\langle\tilde{\alpha}_{l_{0}+1}, \Omega_{2}\right\rangle\right|<\min \left\{\frac{1}{2}, K_{20} \Theta(k) M\left(\omega_{0}, k\right)\right\},\left|\tilde{\alpha}_{l_{0}+1}\right|>1\right\} \\
& >1-\varepsilon_{2}-2 \varepsilon_{2}>\frac{1}{2} .
\end{aligned}
$$

Let us assume that

$$
\begin{aligned}
P\left\{\left|\left\langle\bar{\alpha}_{l_{0}+1}, \Omega_{2}\right\rangle\right| \leqslant K_{20},\left\langle\bar{\alpha}_{l_{0}+1}, \Omega_{1}\right\rangle\right. & \geqslant\left|x_{1}\left\langle\bar{\alpha}_{l_{0}+1}, \Omega_{2}\right\rangle\right| \\
+\frac{1}{4} & \left.>x_{1}\left\langle\bar{\alpha}_{l_{0}+1}, \Omega_{2}\right\rangle+\frac{1}{4}\right\} \geqslant \frac{1}{4} .
\end{aligned}
$$

(If this fails then we have

$$
\begin{aligned}
P\left\{\left|\left\langle\bar{\alpha}_{l_{0}+1}, \Omega_{2}\right\rangle\right|<K_{20},\left\langle\bar{\alpha}_{l_{0}+1}, \Omega_{1}\right\rangle\right. & <-\left|x_{1}\left\langle\bar{\alpha}_{l_{0}+1}, \Omega_{2}\right\rangle\right| \\
& \left.-\frac{1}{4}<x_{1}\left\langle\bar{\alpha}_{l_{0}+1}, \Omega_{2}\right\rangle-\frac{1}{4}\right\}>\frac{1}{4}
\end{aligned}
$$


this can be treated analogously.) Then we have from (2.50), (3.96) and (3.34),

$$
\begin{aligned}
& P\left\{\left\langle\bar{Y}_{1}, \Omega_{2}\right\rangle \geqslant \frac{1}{16}-K_{20} \geqslant \frac{1}{32},\left\langle\bar{Y}_{1}, \Omega_{1}\right\rangle \geqslant x_{1}\left\langle\bar{Y}_{1}, \Omega_{2}\right\rangle+\frac{1}{4}\right\} \\
& >P\left\{\Lambda=l_{0}+1\right\} \\
& \cdot P\left\{\left|\sum_{1<i<l_{0}} \bar{\alpha}_{i}+\sum_{n=1}^{T} \bar{\beta}_{n}, \Omega_{2}\right\rangle \geqslant \frac{1}{16},\right. \\
& \left\langle\sum_{1<i<l_{0}} \bar{\alpha}_{i}+\sum_{n=1}^{T} \bar{\beta}_{n}, \Omega_{1}\right\rangle \geqslant x_{1}\left\langle\sum_{1<i<l_{0}} \bar{\alpha}_{i}+\sum_{n=1}^{T} \beta_{n}, \Omega_{2}\right\rangle, \\
& \geqslant \frac{1}{4} \frac{P\left\{\Lambda=l_{0}+1\right\}}{P\left\{\Lambda=l_{0}\right\}} P\left\{\nu_{1}=T+l_{0},\left\langle\bar{Y}_{1}, \Omega_{2}\right\rangle \geqslant \frac{1}{16},\right. \\
& \left.\geqslant \frac{1}{16} K_{23} T(k) P\left\{J_{1}=1\right\}\left(l_{0}+1\right)^{-1}>K_{20},\left\langle\bar{\alpha}_{l_{0}+1}, \Omega_{1}\right\rangle \geqslant x_{1}\left\langle\bar{\alpha}_{l_{0}+1}, \Omega_{2}\right\rangle+\frac{1}{4}\right\}
\end{aligned}
$$

say, with $K_{24}>0$ independent of $F$ and $k$. We can now complete the proof by a final application of Corollary 2 as before. (3.95) and (3.97) again are versions of (3.8) (resp. (3.9)) with $Z_{1}(1)=\left\langle\bar{Y}_{1}, \Omega_{1}\right\rangle, Z_{1}(2)=\left\langle\bar{Y}_{1}, \Omega_{2}\right\rangle, d_{1}=$ $1 / 32, d_{2}=x_{1}, d_{3}=\frac{1}{4}, p_{3}=\frac{1}{4} K_{23}, p_{4}=K_{24}$. Thus, by (3.10) we have for some $K_{25}<\infty$,

$$
\sum_{r=s}^{2 s} P\left\{\left|\sum_{1}^{r} \bar{Y}_{n}\right| \leqslant 12\right\} \leqslant K_{25}, \quad s>1 .
$$

As in (3.81)-(3.83) the left-hand side of (3.98) is, however, an upper bound for the left-hand side of (3.1). Thus (3.1) holds also in this last case.

Lemmas 4 and 5 prove (3.1) with $k_{5}=\max \left(k_{6}, k_{7}\right), K_{2}=\max \left(K_{14}, K_{19}\right)$. As observed in the beginning of this section, this proves Proposition 1 and our theorem.

\section{REFERENCES}

1. K. L. Chung and W. H. J. Fuchs, On the distribution of values of sums of random variables, Mem. Amer. Math. Soc. No. 6 (1951). MR 12, 722.

2. A. Dvoretzky and P. Erdös, Some problems on random walk in space, Proc. Second Berkeley Sympos. Math. Statist. and Prob. (Berkeley, Calif., 1950), Univ. of California Press, Berkeley, 1951, pp. 353-367. MR 13, 852.

3. H. G. Eggleston, Convexity, Cambridge Univ. Press, Cambridge, 1969. 
4. K. B. Erickson, Recurrence sets of normed random walk in $\mathbf{R}^{d}$, Ann. Probability 4 (1976), 802-828.

5. C. G. Esseen, On the Kolmogorov-Rogozin inequality for the concentration function, $\mathbf{Z}$. Wahrscheinlichkeitstheorie und Verw. Gebiete 5 (1966), 210-216. MR 34 \#5128.

6. - On the concentration function of a sum of independent random variables, $\mathrm{Z}$. Wahrscheinlichkeitstheorie und Verw. Gebiete 9 (1968), 290-308. MR 37 \#6974.

7. W. Hengartner and R. Theodorescu, Concentration functions, Academic Press, New York, 1973. MR 48 \#9781.

8. K. Itô and H. P. McKean, Jr., Diffusion processes and their sample paths, Springer-Verlag, Berlin and New York; Academic Press, New York, 1965. MR 33 \#8031.

9. H. Kesten, The limit points of a normalized random walk, Ann. Math. Statist. 41 (1970), 1173-1205. MR 42 \# 1222.

10. J. V. Uspensky, Introduction to mathematical probability, McGraw-Hill, New York, 1937.

Department of Mathematics, Cornell University, Ithaca, New York 14853 